\title{
Osteología de Hippoglossina macrops (Pleuronectiformes, Paralichthyidae)
}

\author{
Osteology of Hippoglossina macrops (Pleuronectiformes, Paralichthyidae)
}

\author{
Héctor Flores ${ }^{1}$ y Eduardo de la $\mathrm{Hoz}^{2 \dagger}$

\begin{abstract}
${ }^{1}$ Departamento de Acuicultura, Facultad de Ciencias del Mar, Universidad Católica del Norte, Casilla 117, Coquimbo, Chile. hflores@ucn.cl

${ }^{2}$ Laboratorio de Morfología Funcional Animal, Instituto de Biología, Facultad de Ciencias, Pontificia Universidad Católica de Valparaíso, Av. Brasil 2950, Valparaíso, Chile
\end{abstract}

\begin{abstract}
An osteological description of Hippoglossina macrops, a flounder found in the Southeast Pacific, is presented. Specimens between 11 and $32 \mathrm{~cm}$ of total length, captured in the area of Valparaíso and Tomé (Chile) were analyzed. Observations were carried out from dissected specimens previously preserved in formalin and cleared and stained specimens for bone and cartilage. The osteological features of those specimens were then compared to $H$. montemaris. In general, the Hippoglossina macrops osteology have the features given for the suborder Pleuronectoidei, showing diagnostic characters of Paralichthyidae, such as a supra occipital crest inclined towards the nadiral side; metapterygoid related to the upper edge of the quadrate by cartilage and to the rear end by the hyosimplectic cartilage; subopercle with a subsequent upward extension presenting on their sides not ossified surfaces which give uniformity to the bone; fourth abdominal bone in its portion shows a fusion of the first two anal pterygiophores, are among the most important ones. Within the Paralicthyidae family, $H$. macrops it shares characteristics with Pseudorhombus and Paralichthys. $H$. montemaris specimens do not show morphological differences with $H$. macrops, which questioned the validity of the species.
\end{abstract}

Key words: Fishes, flounder, skeleton

Resumen.- Se efectúa una descripción osteológica del lenguado Hippoglossina macrops, presente en el Pacífico suroriental. Se analizaron ejemplares capturados en la zona de Valparaíso y Tomé (Chile), de longitudes totales entre 11 y $32 \mathrm{~cm}$. Las observaciones fueron realizadas a partir de disecciones de ejemplares conservados en formalina y de especímenes coloreados para huesos y cartílago. Se comparó las descripciones osteológicas con ejemplares de H. montemaris. En general, la osteología de Hippoglossina macrops posee los caracteres dados para el suborden Pleuronectoidei, presentando caracteres diagnósticos de Paralichthyidae, siendo los más importantes: la cresta del supraoccipital inclinada hacia el lado nadiral; metapterigoides relacionado con el borde superior del cuadrado por cartílago y en su extremo posterior con el cartílago hiosimpléctico; subopercular con prolongación ascendente posterior presentándose a sus lados superficies no osificadas que dan uniformidad al hueso; cuarto hueso abdominal en su porción presenta fusión de los dos primeros pterigióforos anales. Dentro de la familia Paralichthyidae, H. macrops comparte caracteres de Pseudorhombus y Paralichthys. Los especímenes de $H$. montemaris, no muestran diferencias morfológicas con $H$. macrops, dudando sobre la validez de esta especie.

Palabras clave: Peces, lenguado, esqueleto

\section{INTRODUCCIÓN}

Los peces del orden Pleuronectiformes son peces comprimidos con asimetría cefálica, es decir sus ojos se ubican a un solo lado de la cabeza, debido a su adaptación evolutiva al habitat bentónico que constituiría un grupo monofilético (Chapleau 1993, Hensley 1997). Las larvas son simétricas y la asimetría se desarrolla durante la ontogenia y caracteriza la fase de metamorfosis (Bertin \& Arambourg 1958). Otras características morfológicas de los adultos distinguibles son: lado oculado (zenital) pigmentado y ciego (nadiral) sin pigmento; boca protráctil y relativamente asimétrica; aletas generalmente sin espinas, la dorsal y la anal son de base larga, la caudal es redondeada; ausencia de vejiga gaseosa. La asimetría cefálica involucra preferentemente la zona anterior a la fosa hiomandibular (Chabanaud 1936, Woolcott et al. 1968, Futch et al. 1972), región íntimamente relacionada con las estructuras que participan en la captura de presas.

${ }^{\dagger}$ Fallecido en 1997 
Las familias del orden están bien caracterizadas y concuerdan con la clasificación tradicional (Eschmeyer 1998, Nelson 2006) y con las de enfoque filogenético (Greenwood et al. 1966, Chapleau 1993, Chanet 1997, Hoshino \& Amaoka 1998). En Chile se han descrito 24 especies de Pleuronectiformes (De Buen 1961, Pequeño 1989, 1997), de las cuales tres corresponden a Hippoglossina: H. mystacium, H. macrops e H. montemaris. Las dos últimas son especies muy semejantes entre sí (Pequeño \& D’Ottone 1987), cuya diferencia se basa principalmente en caracteres merísticos y morfométricos, tales como altura máxima y grosor del cuerpo, longitud de la cabeza, cresta interorbitaria (fuerte, saliente que se puede prolongar por detrás de los ojos), branquispinas en la rama superior del primer arco branquial, entre otras características y proporciones. Por otra parte, De Buen (1961) ha planteado que ambas especies habitarían ambientes distintos, $H$. macrops lo haría en los fondos medios de la planicie continental e $H$. montemaris en aguas someras cercanas a la costa, sin embargo, intentos posteriores de recolección y registro de $H$. montemaris han sido infructuosos para efectos de comparación.

Los estudios osteológicos constituyen la base para definir los grandes grupos de peces (Greenwood et al. 1966, Monod 1968, Cervigón 1980), aportando antecedentes para estudios filogenéticos (Chapleau 1993, Hoshino 2001), tanto en especies actuales como extintas (Chanet 1994, 1995, 1996a, 1996b), siendo de utilidad para diferenciación de especies (Kong \& Iratchet 1995, Díaz de Astarloa 2005, Bemvenuti 2005, Dyer 2006), ratificar la pertenencia a un determinado taxa (De la Hoz \& Tosti-Croce 1981), para establecer guías de identificación de especies (Falabella et al. 1995, Deli Antoni et al. 2008) y para comprender las relaciones morfo-funcionales de las estructuras óseas (De la Hoz et al. 1994, De la Hoz 1995, Cancino \& Burgos 2009).

Paralichthyidae es reconocida con el estatus de familia por Amaoka (1969), caracterizándose por presentar ojos generalmente en el lado izquierdo y separados por una cresta ósea; dos orificios nasales a cada lado; el borde del preopérculo libre; aletas sin espinas; aletas dorsal y anal no fusionadas con la caudal; ambas aletas pectorales con 5 a 6 radios; base de ambas aletas pélvicas cortas y casi simétricas; aleta caudal con 17 a 18 radios de los cuales 11 a 13 son ramificados y una línea lateral presente en ambos lados (Hensley 1995, Amaoka \& Hensley 2001).

Hensley \& Ahlstrom (1984) en base a caracteres morfológicos, merísticos y osteológicos postulan que Paralichthyidae estaría estructurada por agrupaciones de especies que conformarían distintos grupos tipos, donde cada grupo podría estructurar una línea filética. Estos grupos serían Cyclopsetta, Pseudorhombus y Paralichthys, determinados por estudios de cariotipo y antecedentes moleculares (Berendzen \& Dimmick 2002, Pardo et al. 2005, Azevedo et al. 2008).

El género Hippoglossina es incluido en el grupo de especies de Paralichthys y a modo de clarificar la posición de Hippoglossina macrops Steindachner, 1876 en la familia Paralichthyidae, se realizó una descripción osteológica que permite complementar los actuales caracteres descritos para esta especie y su relación con la especie nominal $H$. montemaris.

\section{Material y Métodos}

Se trabajó con 37 ejemplares de Hippoglossina macrops capturados de la pesca de arrastre comercial, 25 ejemplares fueron de Valparaíso (11 a $25 \mathrm{~cm}$ de longitud total) y 12 ejemplares de Tomé (13 a $32 \mathrm{~cm})$. Para identificar los especímenes de $H$. macrops, se utilizaron las descripciones dadas por Norman (1934) y De Buen (1961). Posteriormente se analizaron dos ejemplares cuyos caracteres diagnósticos coincidieron con los dados para H. montemaris (6 branquispinas primer arco branquial, cresta interorbitaria poco saliente, color lado oculado café pálido).

Las observaciones y descripciones osteológicas se realizaron en el Laboratorio de Morfología Funcional Animal de la Pontificia Universidad Católica de Valparaíso. Se efectuaron disecciones de ejemplares conservados en formalina y otros coloreados para huesos y cartílago, según la técnica propuesta por Taylor \& Van Dyke (1985). Para la confección de los esquemas se utilizó un microscopio estereoscópico binocular Wild M-5, provisto de cámara clara. Se visualizaron la disposición y relaciones del esqueleto axial a través de una radiografía obtenida de un ejemplar. Los esquemas se dibujaron con tinta y el significado de las abreviaturas se indica en el Anexo 1.

\section{Resultados}

Hippoglossina macrops se caracteriza por presentar a nivel del neurocráneo y esplancnocráneo una asimetría ósea, que involucra preferentemente a las estructuras ubicadas por delante de la fosa de articulación del hiomandibular (Figs. 1 y 2). Desde ese sector hacia atrás, la especie cumple con la simetría propia de todos los teleósteos. 


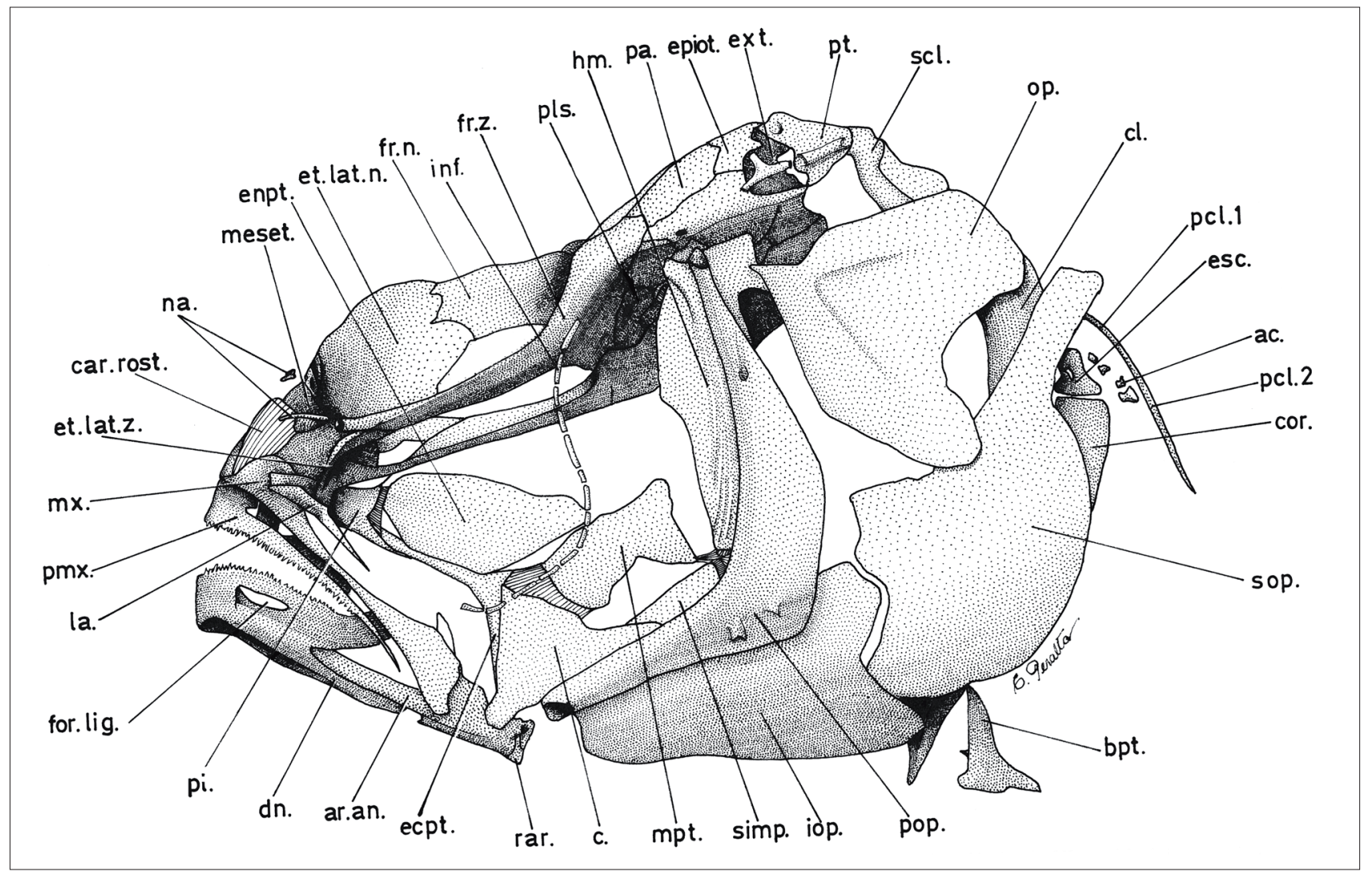

Figura 1. Hippoglossina macrops. Vista lateral izquierda del cráneo / Hippoglossina macrops. Left lateral view of cranium

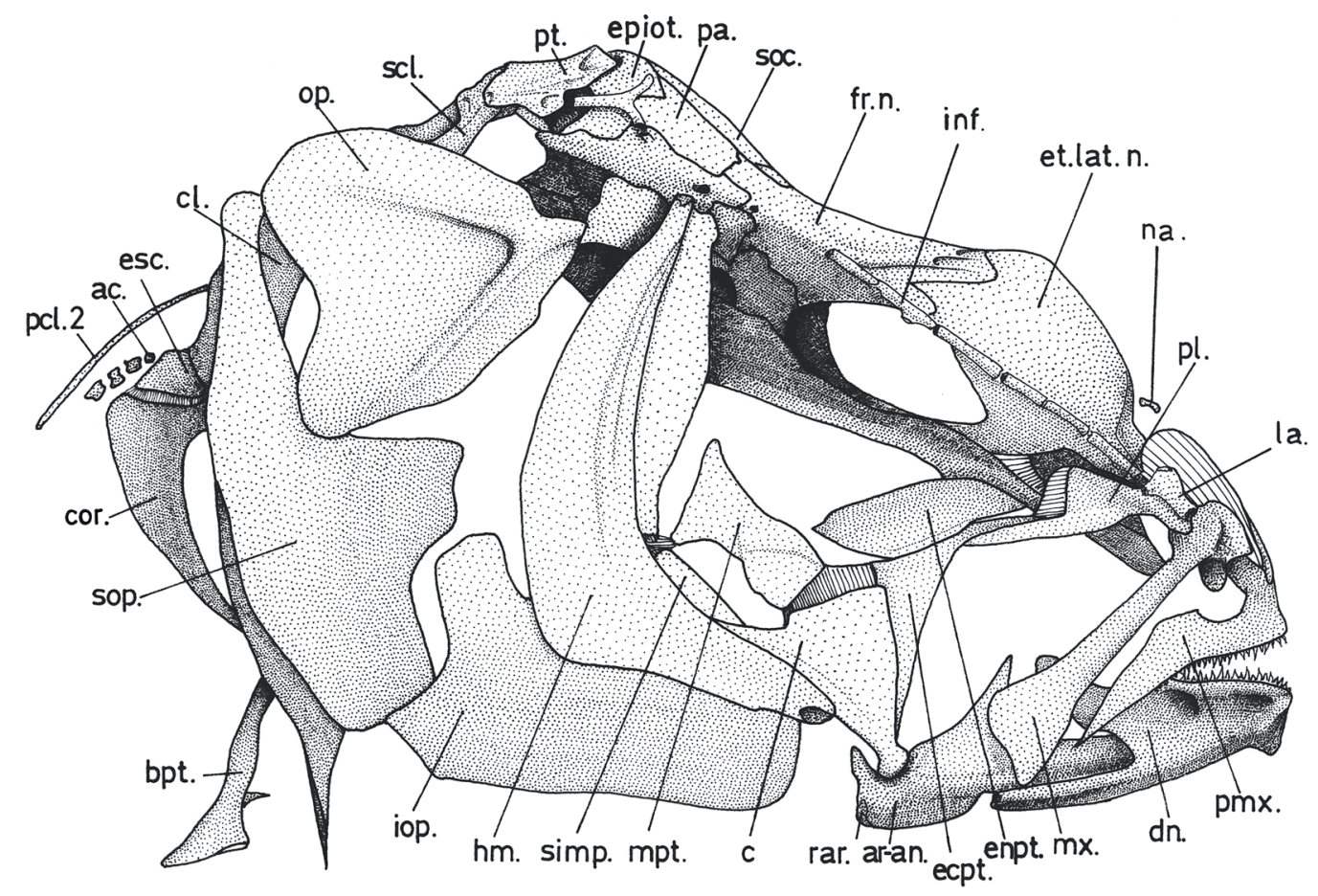

Figura 2. Hippoglossina macrops. Vista lateral derecha del cráneo / Hippoglossina macrops. Right lateral view of cranium 


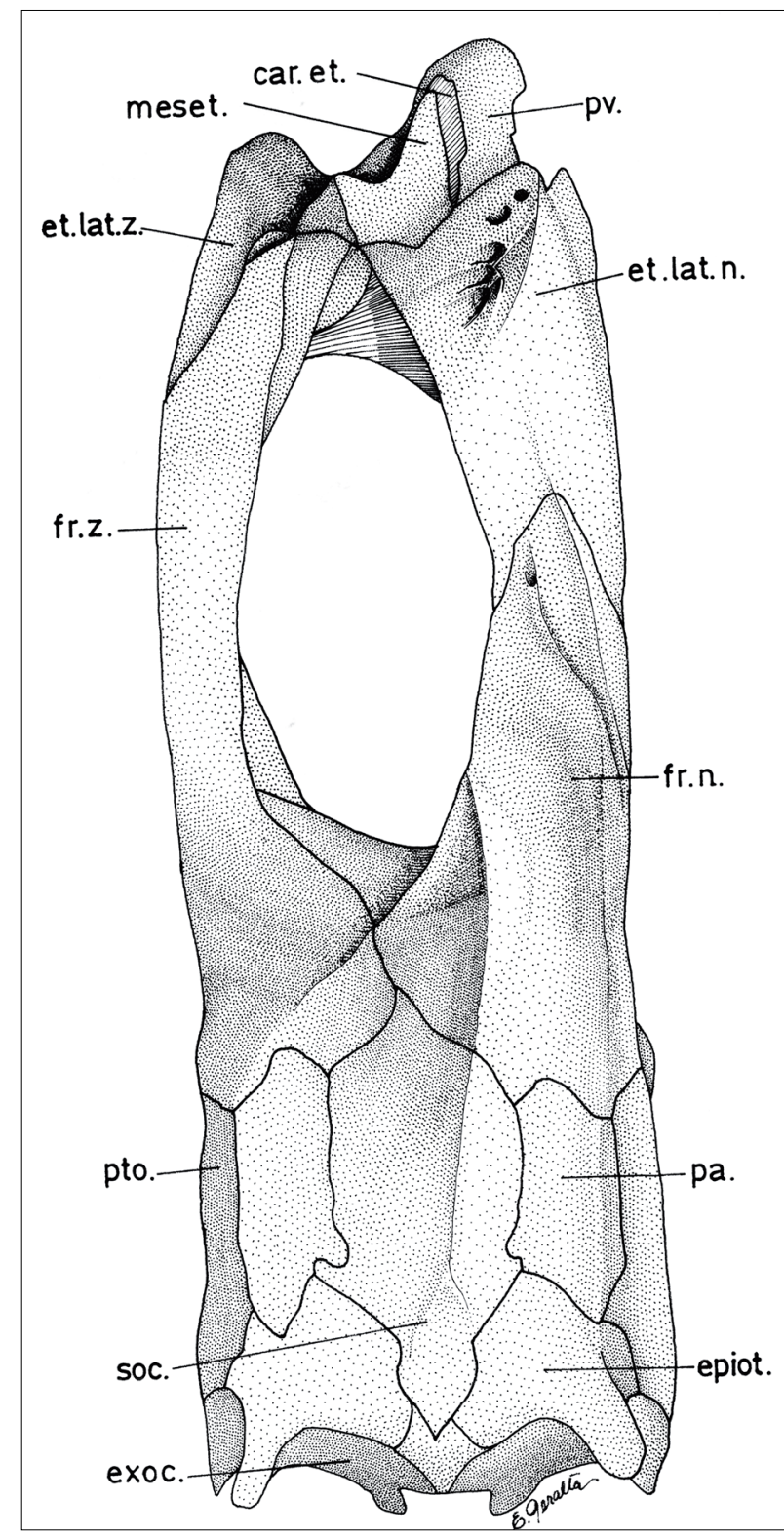

Figura 3. Hippoglossina macrops. Vista dorsal del neurocráneo / Hippoglossina macrops. Dorsal view of neurocranium

El neurocráneo de $H$. macrops presenta una marcada torsión en la región etmoidal, cuya parte dorsal está inclinada hacia el lado izquierdo (oculado, zenital), mientras la parte ventral presenta inclinación hacia el lado derecho (ciego, nadiral) (Figs. 3 y 4); en general aparece bien osificado, exceptuando la región anterior donde persiste un cartílago etmoidal notorio (Figs. 5 y 6).

El prevómer (vómer de Woolcott et al. 1968 y Chabanaud 1936) es edentado, presentando dos alas desarrolladas asimétricamente (Fig. 4). Los etmoides laterales (=prefrontal + paretmoides de Gregory 1951;

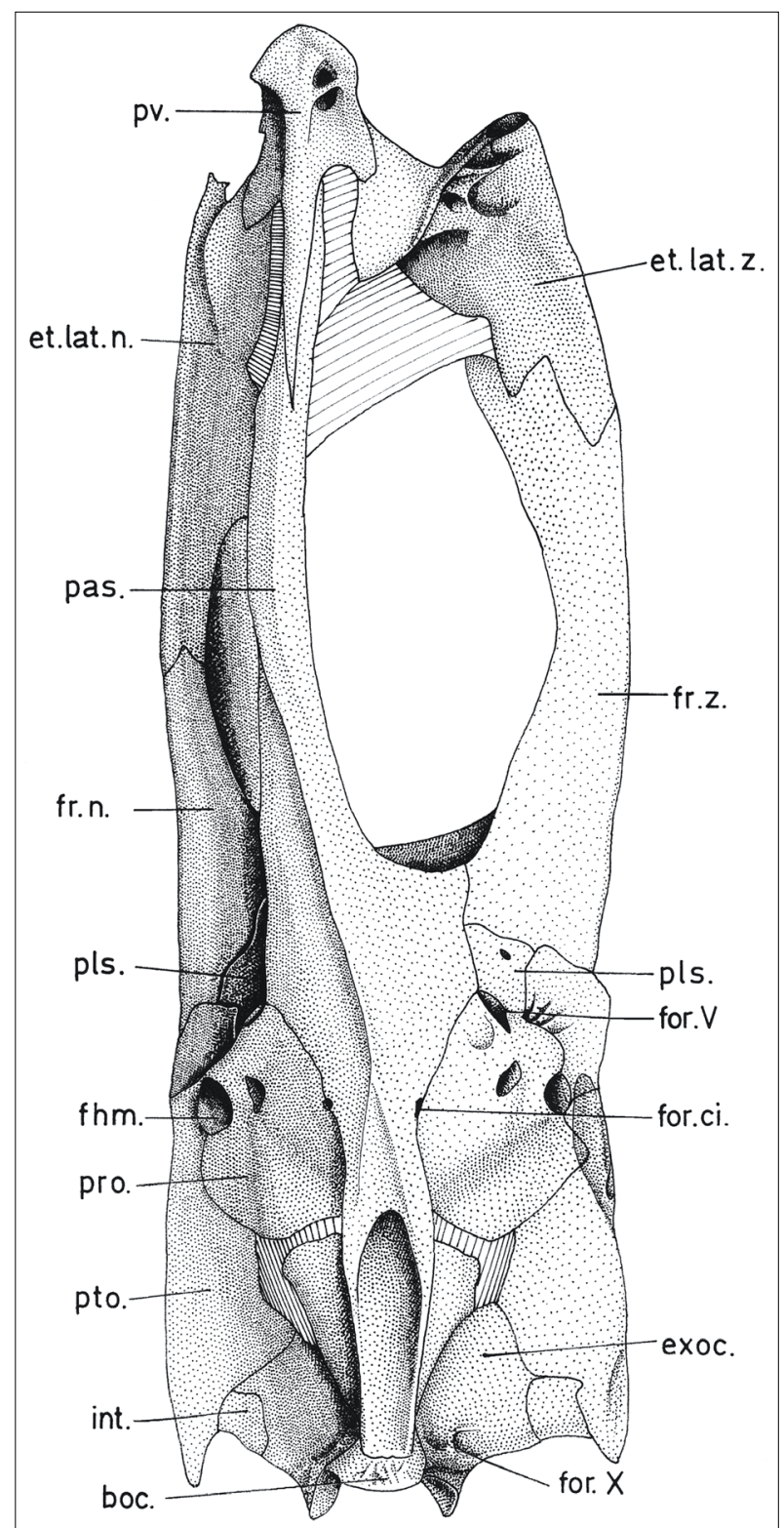

Figura 4. Hippoglossina macrops. Vista ventral del neurocráneo / Hippoglossina macrops. Ventral view of neurocranium

$=$ pleuretmoides de Chabanaud 1936; = prefrontal de Woolcott et al. 1968) son los elementos óseos con mayor asimetría (Figs. 1, 2, 4 y 5).

El mesetmoides (=dermetmoides de Gregory 1951, Woolcott et al. 1968) presenta un borde anterior dirigido hacia el cartílago etmoidal, formando una cresta que sirve para el deslizamiento del cartílago rostral durante la protrusión de las mandíbulas superiores (Figs. 5 y 6).

Los frontales son muy asimétricos, el izquierdo es de mayor longitud, con dos tercios anteriores en forma 


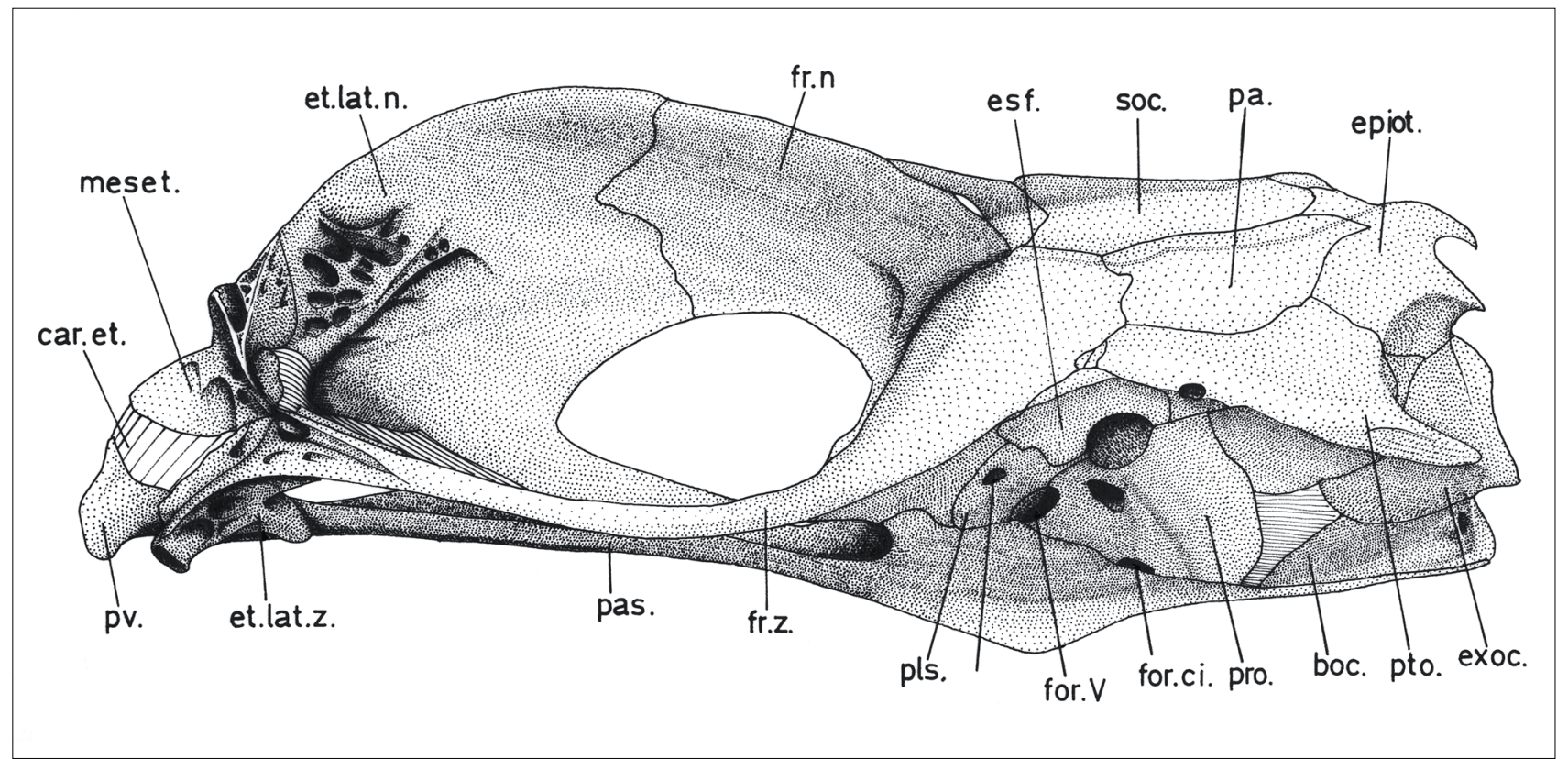

Figura 5. Hippoglossina macrops. Vista lateral izquierda del neurocráneo / Hippoglossina macrops. Left side view of the neurocranium

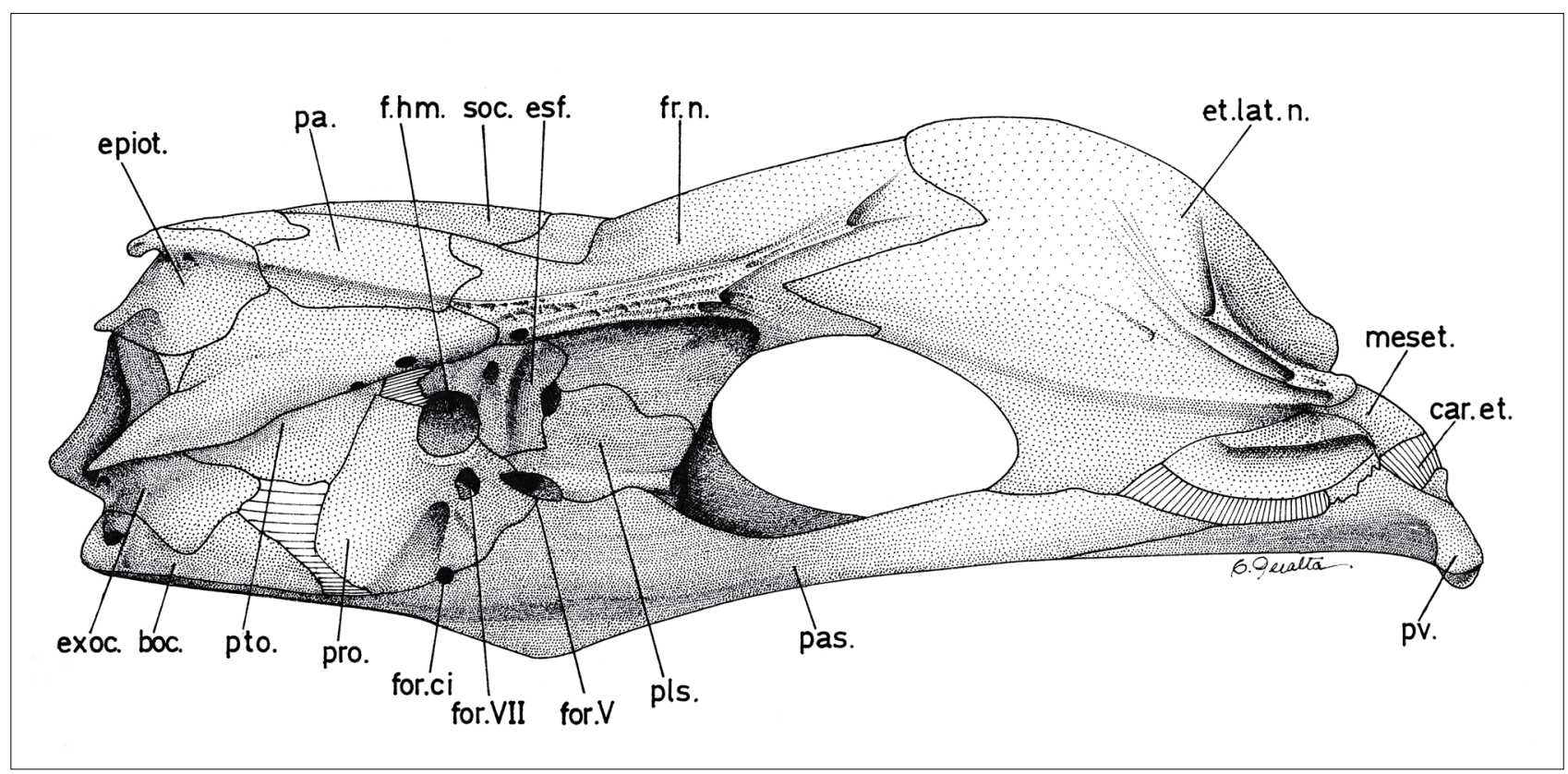

Figura 6. Hippoglossina macrops. Vista lateral derecha del neurocráneo / Hippoglossina macrops. Lateral right view of the neurocranium

de tubo (canal sensorial supraorbital) curvado (Figs. 1, 3,4 y 5), es la única estructura ósea que separa ambos ojos y constituye la cresta interorbitaria que se extiende por detrás de los ojos. El frontal derecho es enteramente laminar, alcanzando anteriormente sólo hasta la mitad de la órbita (Figs. 1, 3, 5 y 6).
La amplia lámina posterior que presenta el etmoides lateral derecho y que articula con el frontal del mismo lado corresponde a lo que Hoshino (2006) denomina "blind side bridge". La región ótica y basicraneal es simétrica (Figs. 3, 4 y 7). Los huesos esfenótico y proótico forman la fosa articular hiomandibular (Figs. 5 y 6), mientras que la concavidad ventral del pterótico articula con el borde 


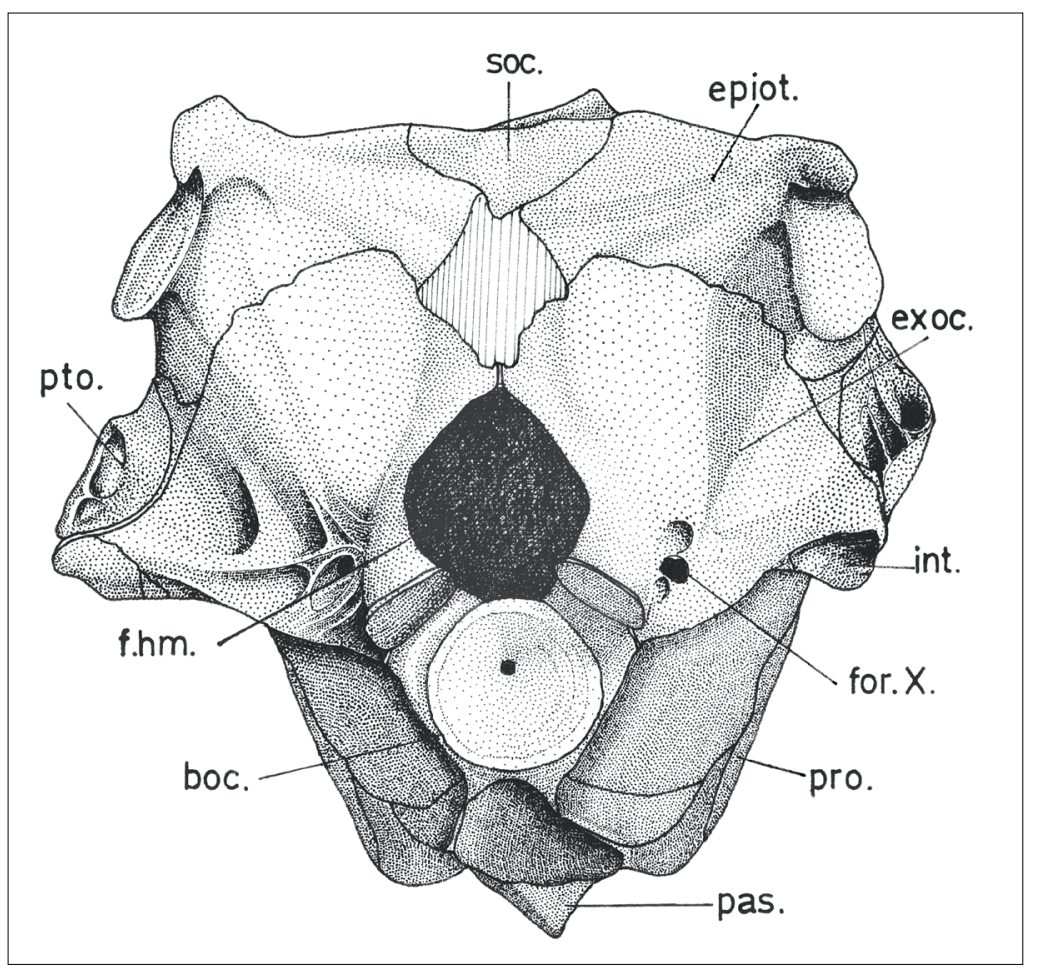

Figura 7. Hippoglossina macrops. Vista posterior del neurocráneo / Hippoglossina macrops. Posterior view of neurocranium

postero dorsal del hiomandibular como segundo punto de unión de este elemento óseo al neurocráneo.

Los exoccipitales forman gran parte del "foramen magnum" y se unen en la línea media superior. Presentan gran desarrollo de sus cóndilos articulares con la columna vertebral, los cuales presentan una inclinación mesial, siendo el basioccipital el elemento óseo que conforma el "foramen magnum" en su parte inferior (Fig. 7).

El intercalar, hueso superficial de membrana, está localizado entre el ala lateral del pterótico y el exoccipital, presentando un fuerte ligamento para articular con la rama ántero-inferior del postemporal (Fig. 4). El supraoccipital presenta una cresta inclinada hacia el lado derecho, continuando anteriormente en el frontal nadiral, que sirve de base para la implantación de los 9 primeros pterigióforos dorsales (Fig. 3).

La serie de huesos infraorbitarios se modifica y difiere en el número y posición de sus elementos óseos entre el lado izquierdo y derecho (Figs. 1 y 2). El frontal derecho, hueso que aloja el canal supraorbital del sistema sensorial lateral, posee dos aperturas anteriores: la más dorsal termina en dicha zona y la inferior comunica el sistema lateral, por una serie de 6 infraorbitarios tubulares, con el lacrimal y por éste con el nasal derecho (Fig. 2). En el lado izquierdo, es el frontal el que comunica el sistema lateral con el nasal, mientras que una serie de 15 a 18 infraorbitarios tubulares, está conectada posteriormente al frontal y anteriormente con el ectopterigoides a nivel del vértice superior del cuadrado (Fig. 1). El lacrimal izquierdo presenta orificios para el paso del canal sensorial, participando además en funciones de protracción de las mandíbulas superiores.

Al igual que el neurocráneo, se aprecia una asimetría del esplancnocráneo que afecta principalmente su parte anterior, especialmente a los huesos palatinos, premaxilares, maxilares, metapterigoides y endopterigoides (Figs. 1 y 2). El dentario presenta una serie de dientes cónicos y curvados hacia el interior dispuestos en su borde superior, similares a los que poseen las premaxilas. En la mitad anterior existe un gran foramen en cuyo borde anterior se fija el ligamento dentario-maxilar. Este foramen permite alojar la base voluminosa de este ligamento cuando las mandíbulas están cerradas (Figs. 1 y 2). El articular-angular, por su porción anterior, se relaciona con el dentario por una depresión interna de éste. Posee una rama ascendente que sirve de inserción a los ligamentos lacrimal-articular-angular y maxilar-articular-angular, ambos importantes, pues se fija el músculo aductor mandibular. En su borde interno se presenta el coronomekeliano (Figs. 8 y 9). 


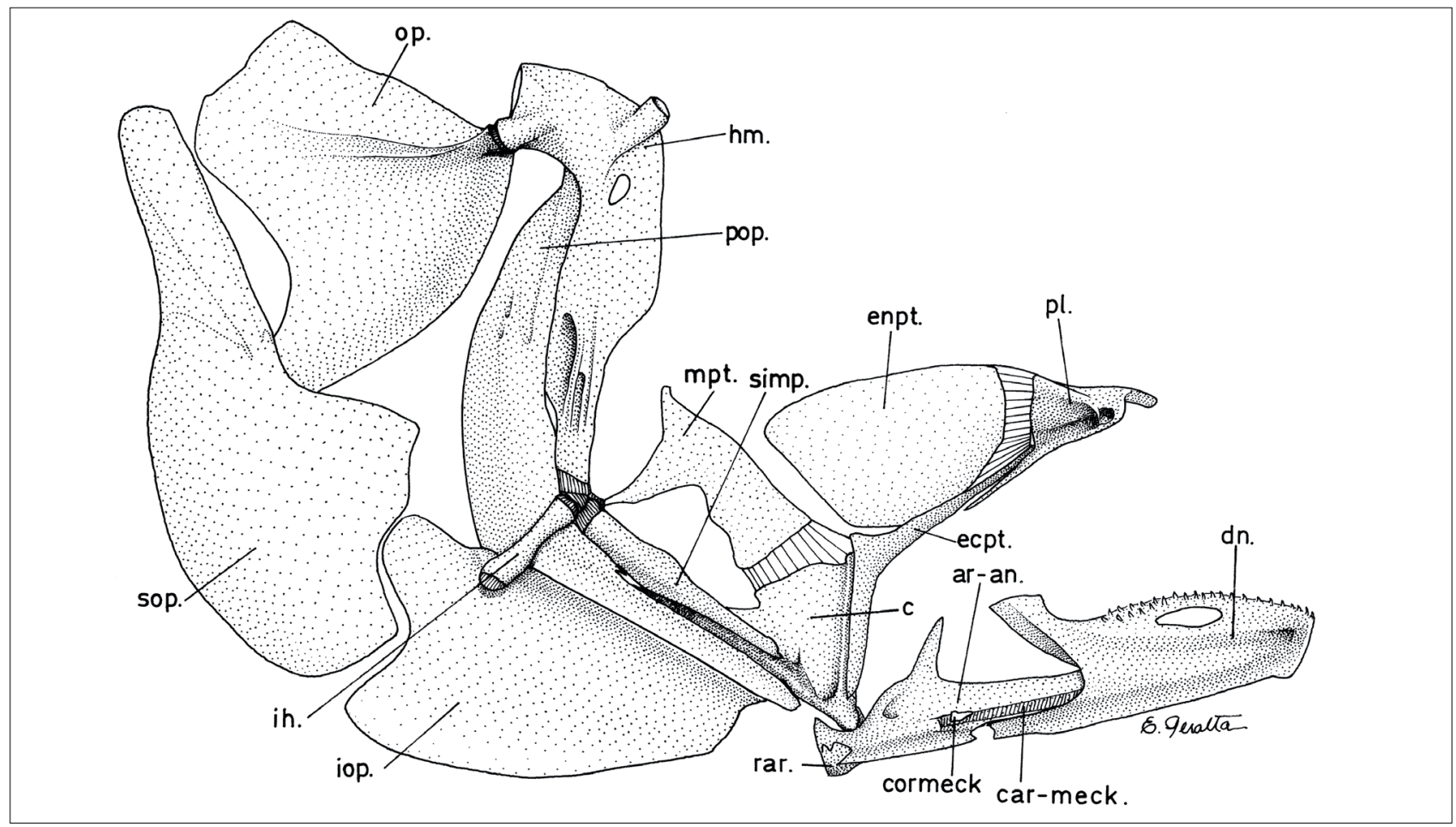

Figura 8. Hippoglossina macrops. Vista izquierda interna del esplancnocráneo / Hippoglossina macrops. Left internal view of splanchnocranium

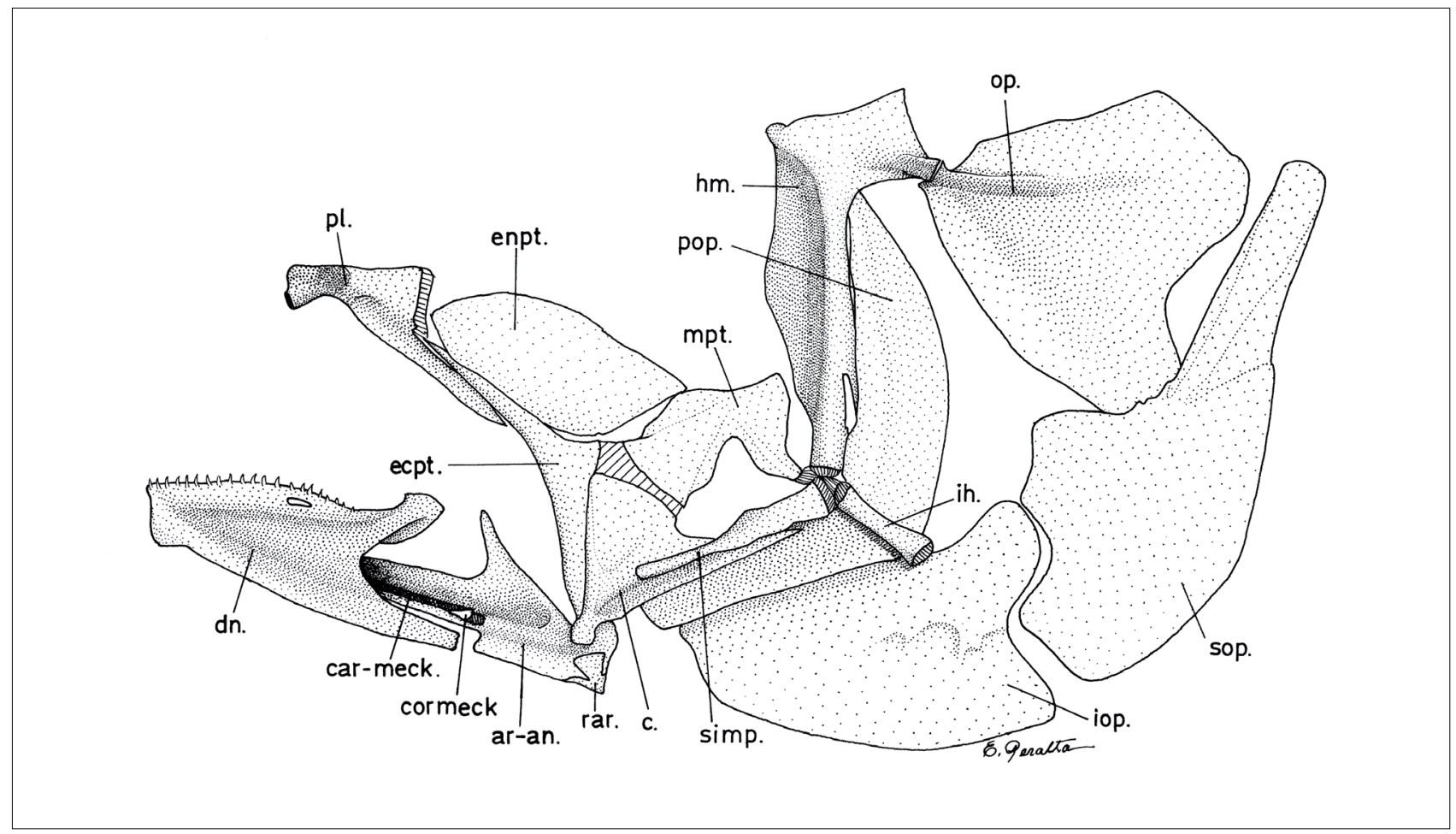

Figura 9. Hippoglossina macrops. Vista derecha interna del esplancnocráneo / Hippoglossina macrops. Right internal view of splanchnocranium 


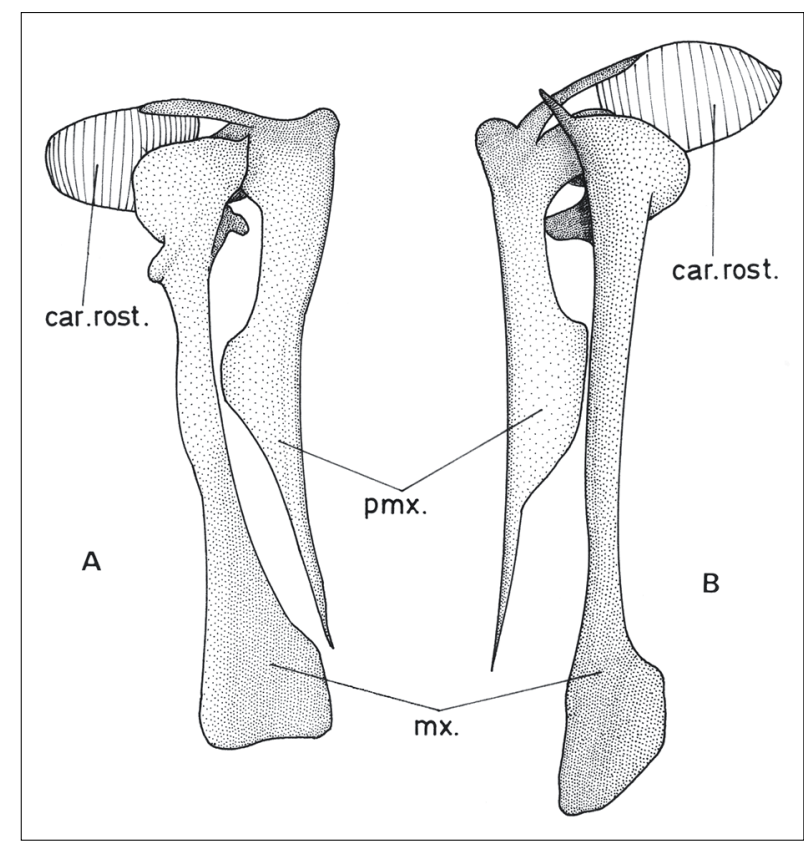

Figura 10. Hippoglossina macrops. Vista lateral de la mandíbula superior. A) lado derecho, B) lado izquierdo / Hippoglossina macrops. Lateral view of upper jaw. A) right side, B) left side

Los huesos premaxilares (Figs. 1, 2 y 10) presentan procesos articulares para maxila y mesetmoides, la dentadura recorre casi todo su borde anterior, dispuesta a una serie de dientes cónicos, curvados internamente. Los huesos maxilares se relacionan con los premaxilares por fuertes ligamentos internos. La rama ascendente del palatino sobremonta la maxila fijándose por un ligamento (Figs. 1 y 2). Articulan con el prevómer a través de meniscos articulares y por cuatro ligamentos al cartílago rostral en su parte posterior, presentando una concavidad para deslizarse en el mesetmoides y cartílago etmoidal, así ambos contribuyen a la protracción de las premaxilas.

El suspensorio se fija anteriormente al neurocráneo a través del palatino con el ala del etmoides lateral. El palatino, por la cara interna de su proceso anterior, está articulado con los premaxilares mediante un ligamento. Por su rama descendente, en su parte interna está articulado con el ectopterigoides, mientras que con el endopterigoides hay una unión mediante cartílago, sólo en el lado oculado (Figs. 1 y 2). El metapterigoides está relacionado con el borde superior del cuadrado mediante cartílago y en su extremo posterior con el cartílago hiosimpléctico.

El hiomandibular (Figs. 1, 2, 8 y 9) presenta dos procesos articulares relacionados al neurocráneo; el cóndilo anterior se fija en una fosa formada por el proótico y esfenótico, mientras que su borde dorsal se relaciona con una depresión del pterótico. El opercular articula con el hiomandibular por un cóndilo articular sobrepasando al hiomandibular por su lado externo. El extremo inferior posee cartílago que articula con el simpléctico e interhial. El ala anterior es laminada y sirve como base de fijación a fascículos del músculo aductor mandibular.

La porción ascendente del preopercular, está sobre la región articular del hiomandibular y conectado al canal sensorial proveniente de la zona temporal. En la serie opercular, cabe destacar una prolongación ascendente posterior del subopercular, presentándose en sus lados superficies no osificadas que dan uniformidad al borde de dicho hueso (Figs. 8 y 9). Entre la serie opercular y el preopercular existe un espacio membranoso.

En el eje antero-posterior del arco hioideo existen cuatro centros de osificación: ceratohial posterior, ceratohial medio, ceratohial anterior e hipohial (Daget 1964) (Fig. 11), equivalente a epihial, ceratohial, hipohial superior e inferior de otros autores (Gregory 1951, Woolcott et al. 1968, Futch et al. 1972). El ceratohial medio, presenta una depresión y un foramen (Fig. 11). Este orificio y el del ceratohial anterior permiten el paso de la arteria eferente hiodiana. La articulación entre ceratohial medio y posterior es principalmente cartilaginosa con interdigitaciones externas más numerosas en la cara lateral.

Los branquiostegales se disponen por la cara externa de la barra hioidea, el primero por sobre el ceratohial medio, el segundo y tercero en las depresiones de este hueso y los cuatro restantes se ubican a nivel de la articulación ceratohial medio-posterior y sobre éste último.

La estructura de los arcos branquiales (Fig. 12) sigue el patrón de otros Paralichthyidae. El cuarto basibranquial y cuarto faringobranquial serían cartilaginosos. El quinto ceratobranquial presenta una gran placa dentada. La placa faringobranquial 3 está fusionada al faringobranquial 3, mientras que la placa faringobranquial 4 , se ubica sobre el cartílago correspondiente al cuarto faringobranquial. El tercer epibranquial presenta una placa dentada. La rama superior del primer arco branquial presenta 5 branquispinas.

La cintura pectoral (Figs. 13 y 14), presenta dos puntos de articulación con el neurocráneo, mediante el postemporal, que por su zona ascendente articula con el epiótico y por la inferior articula con el intercalar. Presenta un conducto para el paso del canal sensorial postemporal que se continúa hacia adelante por el extraescapular (Figs. 1 y 2), existiendo dos en el lado izquierdo, el más anterior con forma de ' $\mathrm{Y}$ ' y el posterior cilíndrico, 


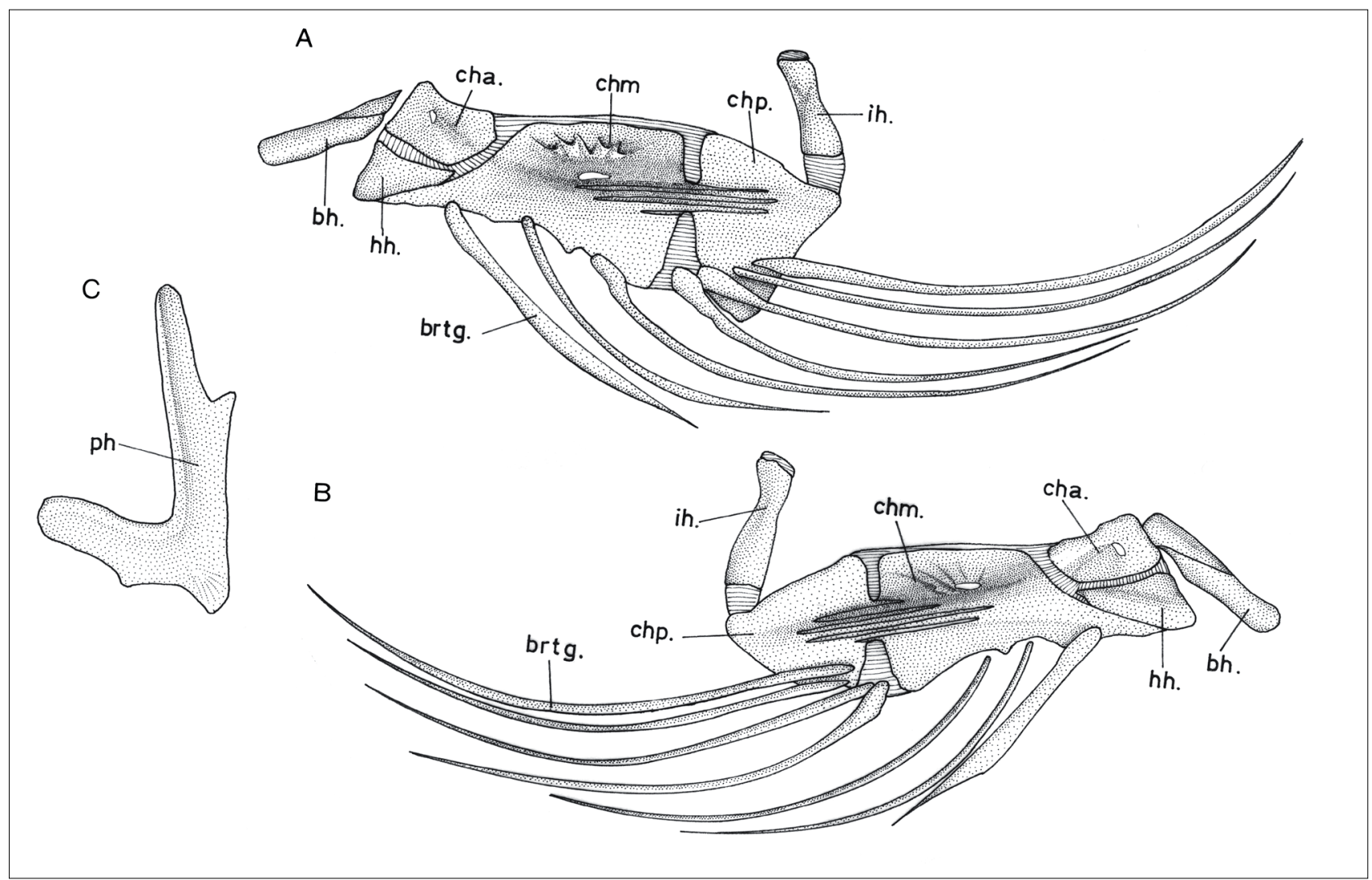

Figura 11. Hippoglossina macrops. Vista lateral. A-B) arco hioídeo y C) parahial / Hippoglossina macrops. Lateral view. A-B) hyoid arch and C) parahyal

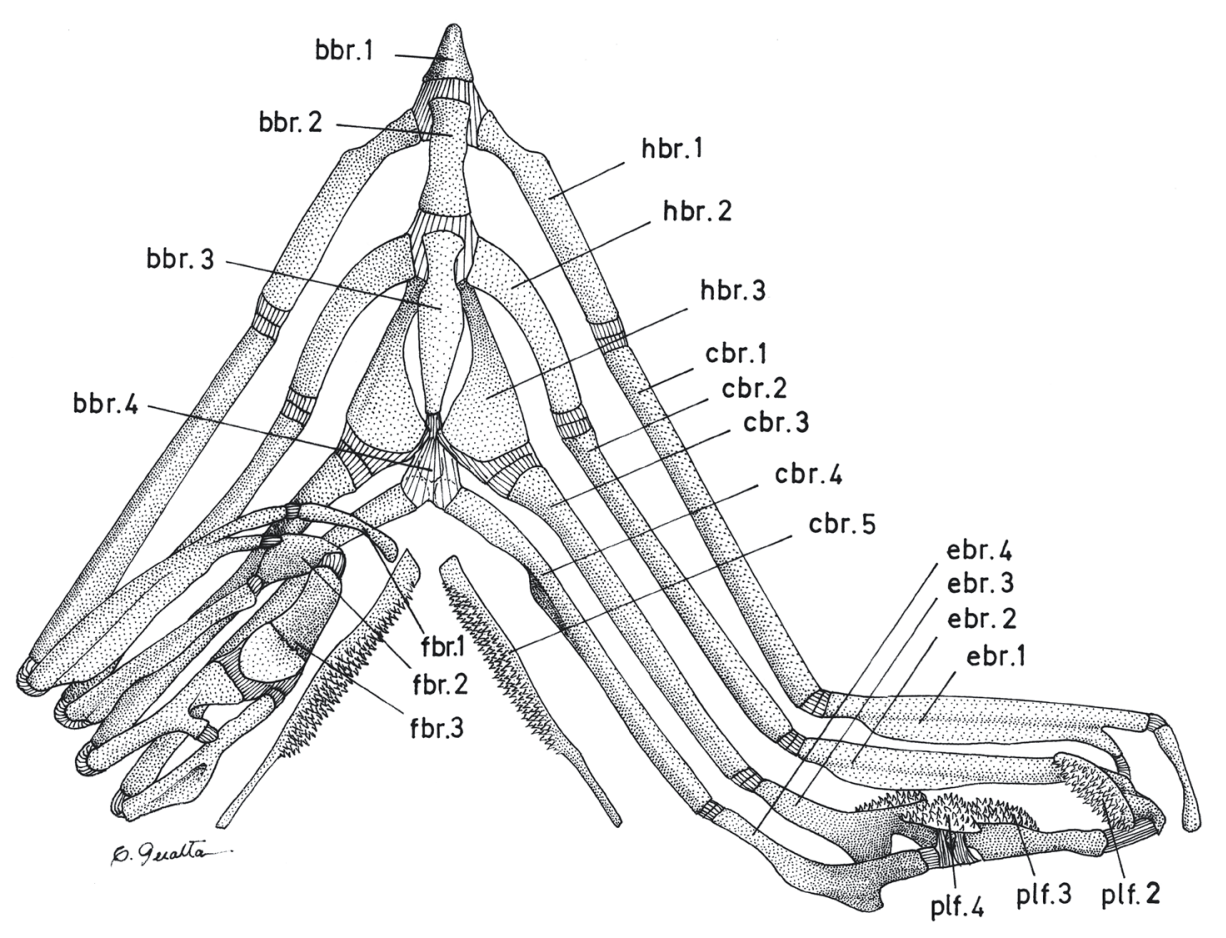

Figura 12. Hippoglossina macrops. Vista dorsal del aparato branquial / Hippoglossina macrops. Dorsal view of branchial apparatus 


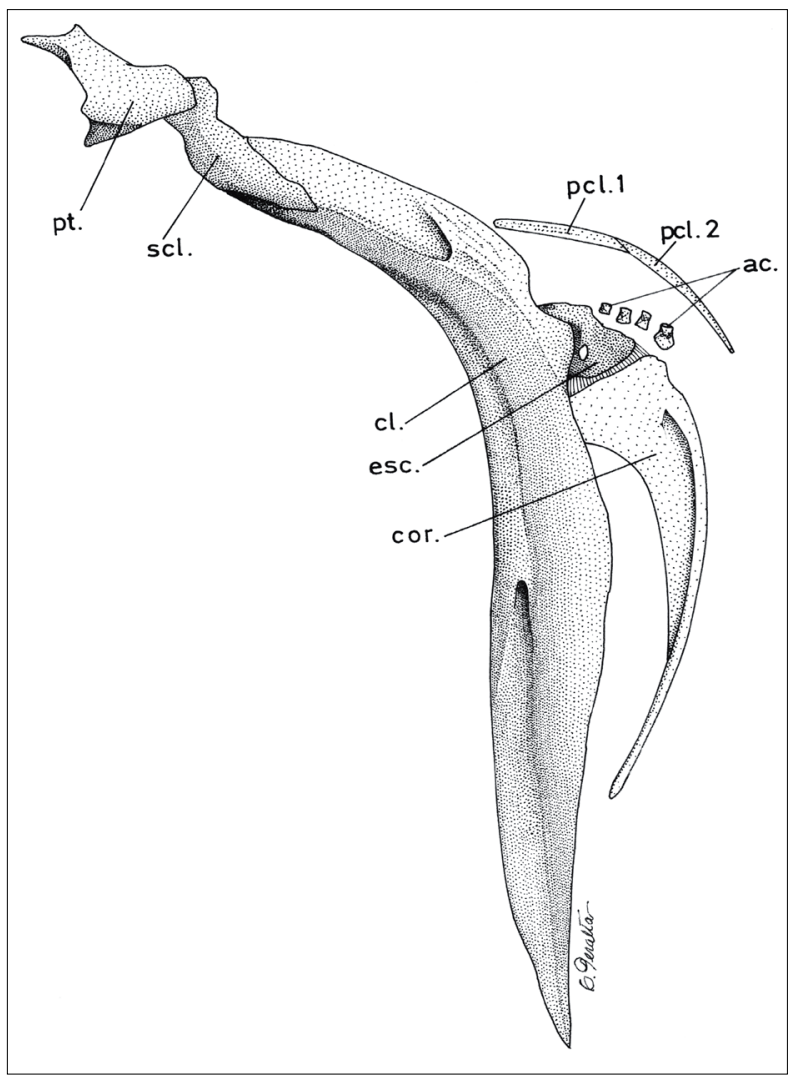

Figura 13. Hippoglossina macrops. Vista lateral izquierda de la cintura escapular / Hippoglossina macrops. Left lateral view of scapular girdle

ambos relacionados con el pterótico, mientras que en el lado derecho encontramos solo uno. Los escapulares se encuentran perforados para el paso de nervios y vasos, y por detrás de estos y del coracoides se ubican cuatro actinostos pectorales. Todos estos huesos son de origen condral. Por detrás de los cleitra y actinostos, se disponen dos postcleitra. Los basipterigios (Fig. 15) se articulan anteriormente en el espacio que dejan la cleitra derecha e izquierda y se adicionan 6 radios en cada uno de ellos.

La columna vertebral consta de 11 vértebras precaudales y 27 caudales incluyendo el urostilo (Figs. 16 y 17). La espina neural del atlas está dispuesta perpendicular al eje del cuerpo vertebral. En las siguientes vértebras, las espinas neurales están inclinadas paulatinamente hacia atrás hasta la cuarta vértebra, diferenciándose estas cuatro primeras en su consistencia. La espina neural de la sexta vértebra está en contacto con la quinta espina neural, la cual está dispuesta perpendicular a su centrum. En las vértebras 7 a 11 (Fig. 18), existe una leve inclinación anterior de las espinas neurales, volviendo a su posición perpendicular en la primera vértebra caudal. Desde la vértebra 12 a la

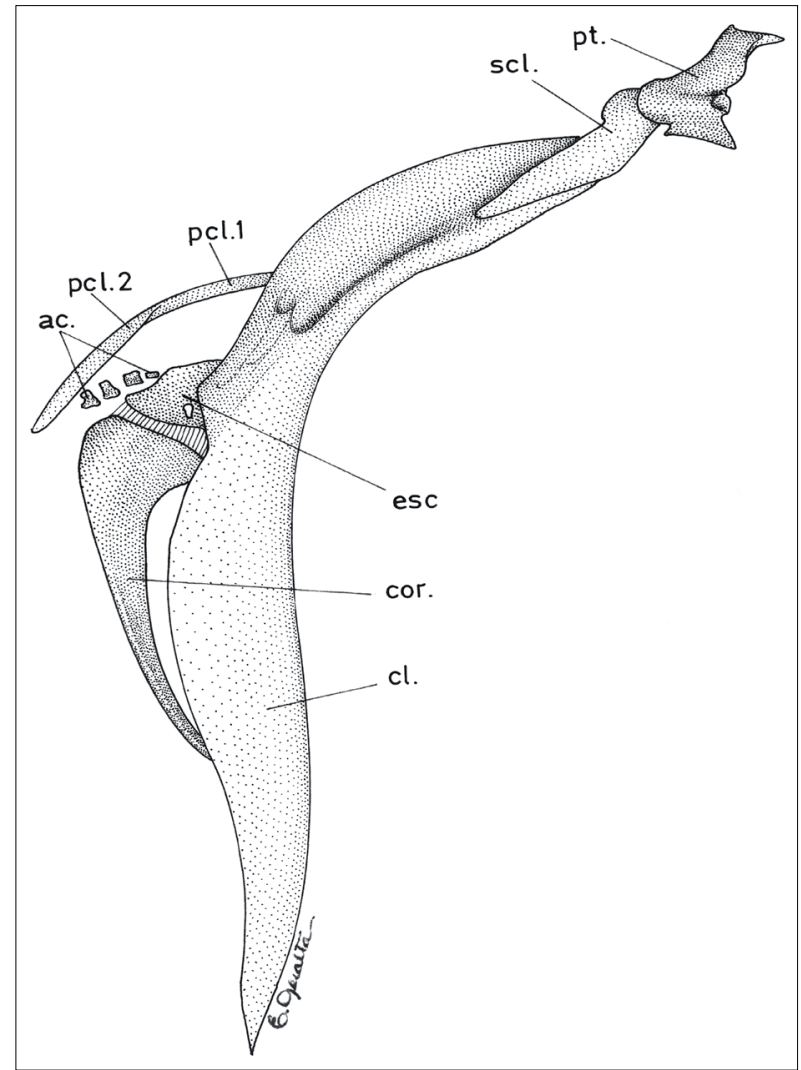

Figura 14. Hippoglossina macrops. Vista lateral derecha de la cintura escapular / Hippoglossina macrops. Right lateral view of scapular girdle

última, las espinas neurales se inclinan secuencialmente en sentido posterior. Hay desarrollo de prezigapófisis y poszigapófisis neurales, destacada fundamentalmente en las vértebras del tronco. Las vértebras 5 a la 11 han desarrollado hemapófisis, cuya base involucra todo el cuerpo vertebral y sus vértices curvados en sentido posterior (Fig. 18).

La primera vértebra caudal, está considerablemente modificada. Su espina hemal es muy larga y profundamente acanalada anteriormente para soportar el hueso abdominal ("abdominal rod"), que cierra posteriormente la cavidad abdominal (Fig. 16). Entre el hueso abdominal y la primera espina hemal se distribuyen diez pterigióforos, donde el extremo distal anterior de este hueso se encuentra fusionado con los dos primeros pterigióforos anales. Las espinas hemales se inclinan en sentido posterior, hacia la última vértebra, no presentando desarrollo de prezigapófisis y poszigapófisis.

A nivel dorsal, los dos primeros pterigióforos dorsales están fusionados, encontrándose nueve pterigióforos anteriores al primer arco neural, los que están insertos sobre el frontal nadiral-supraoccipital. Cada pterigióforo soporta una 


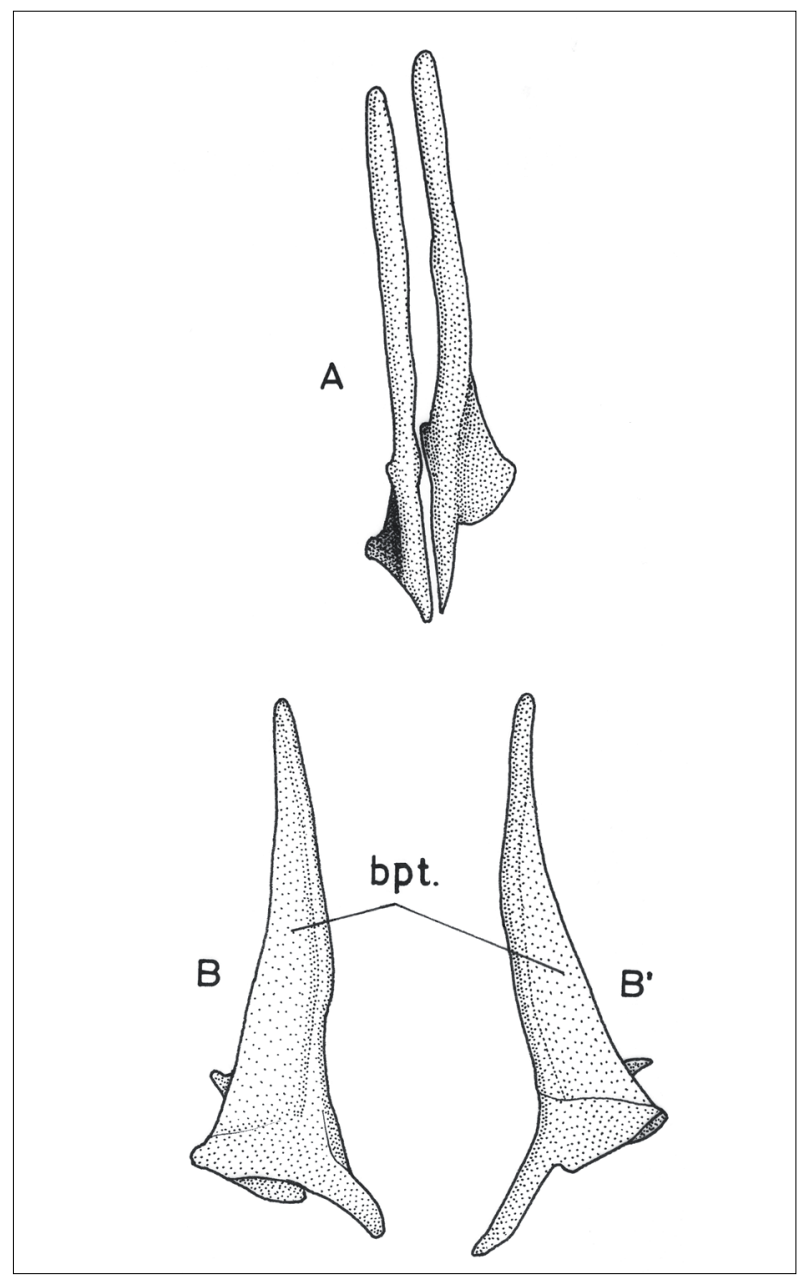

Figura 15. Hippoglossina macrops. Basipterigoides. A) vista posterior, B) vista lateral izquierda y B') vista lateral derecha ( Hippoglossina macrops. Basipterygoid. A) posterior view, B) left lateral view and $\mathrm{B}^{\prime}$ ) right lateral view lepidotrica. En la región abdominal, se detectan dos series de costillas a partir de la segunda vértebra y en toda la región abdominal se presentan las costillas neoneurales, mientras que las costillas epineurales, para la misma zona comienzan en la tercera vértebra (Fig. 17). Las costillas pleurales se desarrollan sólo en las vértebras que poseen hemapófisis (Fig. 18). La penúltima vértebra se encuentra modificada y sus espinas neurales y hemales se elongan y ensanchan para soportar las dos lepidotricas más exteriores (Fig. 19). No hay desarrollo de apófisis transversas.

El esqueleto caudal (Fig. 19) concuerda en sus aspectos fundamentales al descrito para un Perciforme primitivo. La nomenclatura usada es la establecida por Gosline (1961) y Monod (1968). El parhipural es adyacente a la espina hemal de la última vértebra. La placa hipural I articula con la vértebra urostilar, mientras que la placa hipural II sobre la línea media se encuentra fusionada al centrum preural I, ural I y uroneural, formando entre ellas una sola estructura, dorsalmente a ésta se ubica un epural. Hay presencia de 17 radios más un radio procurrente en el extremo de cada lóbulo, en el cual el superior es libre y se conecta con la última espina neural, mientras que el inferior se fusiona con el último radio. La estructura de radios no segmentados, segmentados y ramificados según la propuesta de Hoshino (2001) es: $1+2+7+6+2 \pm 1$.

Los ejemplares que presentaban caracteres diagnósticos de H. montemaris (presencia de 6 branquispinas en el primer arco branquial, una cresta interorbitaria poco saliente y de color café pálido en su lado oculado), no mostraron diferencias morfológicas con H. macrops según el análisis de las estructuras óseas, situación que mantiene la duda sobre la validez de la especie $H$. montemaris.

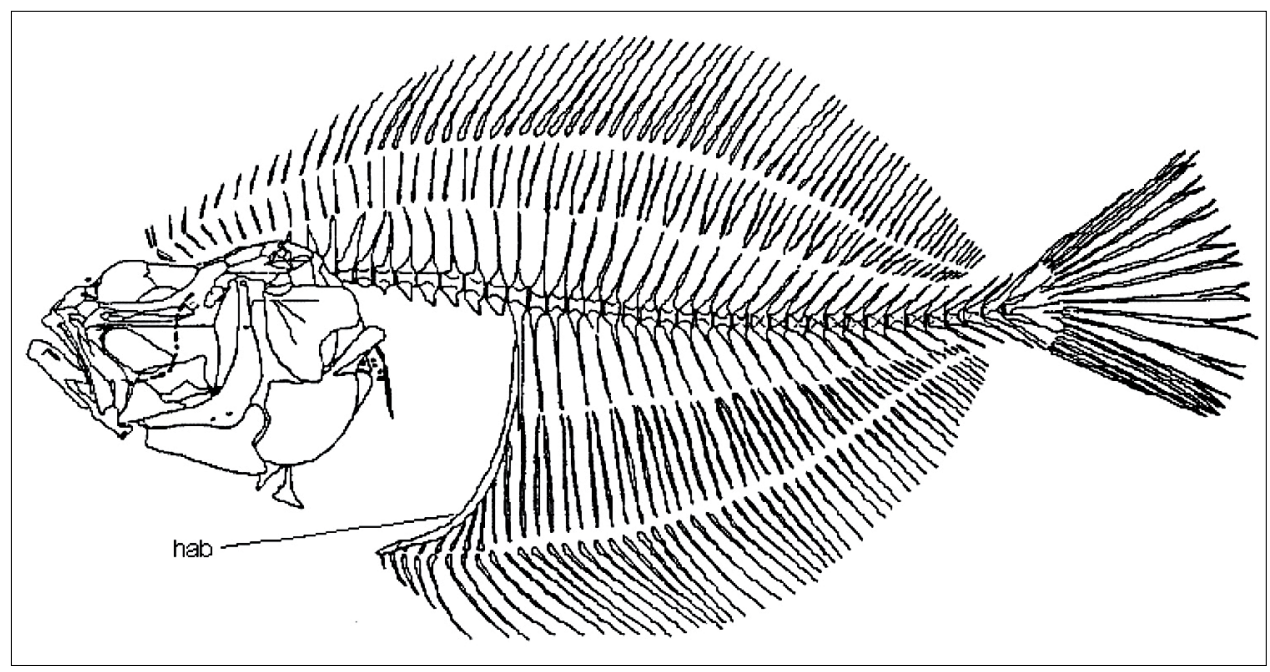

Figura 16. Hippoglossina macrops. Esqueleto axial. Distribución de estructuras y disposición de hueso abdominal (hab) / Hippoglossina macrops. Axial skeleton. Distribution and arrangement of structures of abdominal rod (hab) 


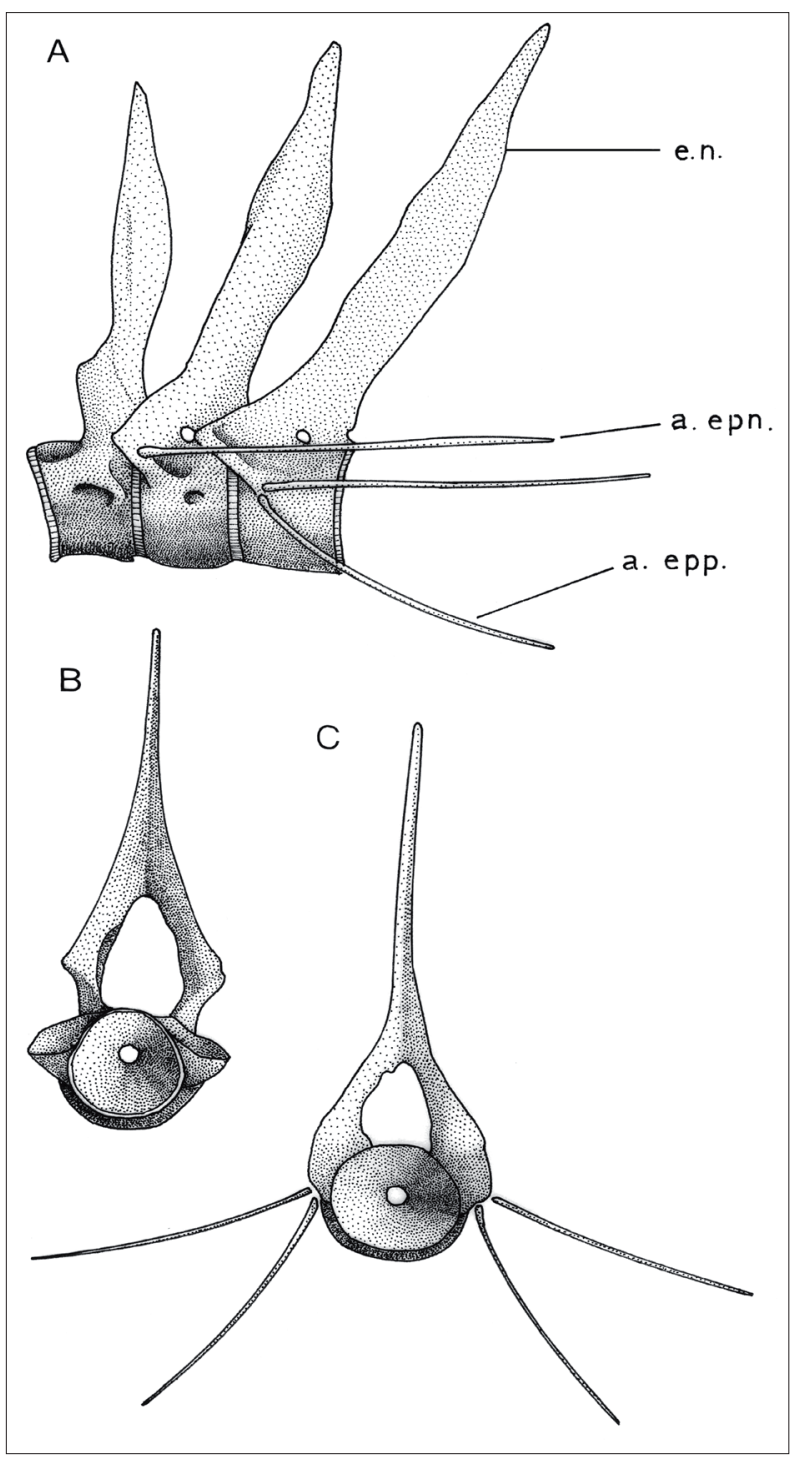

Figura 17. Hippoglossina macrops. Esqueleto axial. A) primeras tres vértebras en vista lateral, B) vista anterior del atlas y C) vista anterior de la tercera vértebra / Hippoglossina macrops. Axial skeleton. A) lateral view of first three vertebrae, B) front view of atlas and C) front view of third vertebra

\section{Discusión}

En Hippoglossina macrops, los pleurosfenoides están separados, al igual que en otros Paralichthyidae como Paralichthys, Tephrinectes y Citharichthys (= pterosfenoides de Hoshino \& Amaoka 1998, Kobelkowsky 2002, Díaz de Astarloa 2005), Mancopsetta (Achiropsettidae) (Evseenko 1996) y Scophthalmus (Scophthalmidae), mientras que en Samaris (Samaridae), Solea (Soleidae) y Cynoglossus (Cynoglossidae) han desaparecido completamente (Chabanaud 1936).
La serie de huesos infraorbitarios no se encuentran presentes en Bothidae (Amaoka 1969), existiendo presencia en los géneros de Paralichthyidae (Hensley \& Ahlstrom 1984, Hoshino \& Amaoka 1998). El patrón estructural de los arcos branquiales de H. macrops es semejante a otros Paralichthyidae como son Paralichthys olivaceus (Hosoyama \& Kawamura 1993), P. isosceles, P. orbignyanus y $P$. patagonicus (Díaz de Astarloa 2005), Citharichthys spilopterus (Kobelkowsky 2002) y Tephrinectes sinensis (Hoshino \& Amaoka 1998).

En H. macrops la estructura de la cintura pectoral corresponde al patrón descrito para Paralichthys (Balart 1985). La columna vertebral, en la forma y disposición de las espinas neurales de sus cuatro primeras vértebras, muestra gran similitud con Paralichthys (Woolcott et al. 1968), especialmente el atlas, que en el caso de Citharichthys spilopterus se fija firmemente a la pared posterior del neurocráneo (Kobelkowsky 2002). No hay desarrollo de apófisis transversas, tal como ocurre en otros Paralichthyidae como Citharichthys, Cyclopsetta, Etropus y Syacium (Hensley \& Ahlstrom 1984, Murakami \& Amaoka 1992)

El extremo distal del hueso abdominal se encuentra fusionado con los dos primeros pterigióforos anales, a diferencia de Paralichthys en que sólo hay fusión del primer pterigióforo (Woolcott et al. 1968). Hippoglossina macrops no presenta huesos intermusculares, tal como reportan Patterson \& Johnson (1995) para Bothus (Bothidae) y Samaris (Samaridae).

En Paralichthyidae el complejo hipural es variable y se reconocen al menos dos patrones de los propuestos por Hensley \& Ahlstrom (1984), el patrón 1 sería para Tephrinectes y Thysanopsetta y el patrón 6 caracterizado por tener los hipurales 1 y 2 fusionados (Placa hipural I), formando un elemento que articula con la superficie posteroventral del urostilo y los hipurales 3 y 4 fusionados, que forman la sección terminal del centrum medio. Sumida et al. (1979) reportan para Hippoglossina stomata la ausencia de epural libre, la explicación de los autores es que sería un carácter específico para esta especie, ya que habría otras especies de este género que si lo poseerían, tal como ocurre con H. oblonga, donde hay diferenciación entre el epural y el quinto hipural (Hensley \& Ahlstrom 1984). En el caso de H. macrops, presenta un solo elemento óseo que corresponde a la fusión del quinto hipural + epural y que también fue reportado para esta especie por Hoshino (2001). 


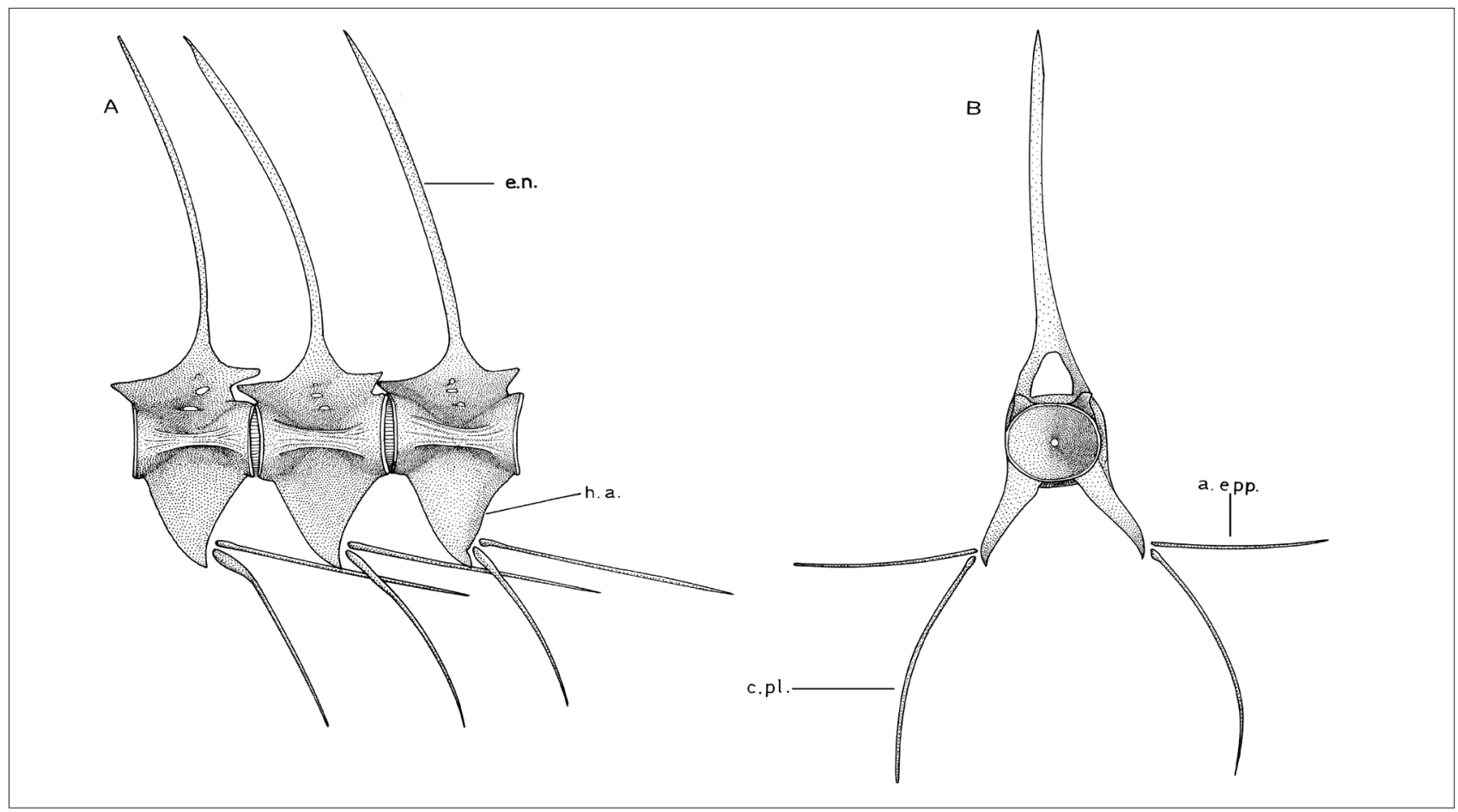

Figura 18. Hippoglossina macrops. Esqueleto axial. A) vista lateral de las vértebras 9 a 11 y B) vista anterior de la vértebra 9 / Hippoglossina macrops. Axial skeleton. A) lateral view of vertebrae 9 to 11 and B) front view of vertebra 9

El grupo Paralichthys propuesto por Hensley \& Ahlstrom (1984) incluye a Hippoglossina macrops, que comparte varios caracteres de tipo osteológico con este grupo, tales como: presencia de costillas epipleurales y pleurales; ausencia de huesos intermusculares; presencia de postcleitrum; ausencia de dientes vomerianos y de supramaxilar; vértebras sin apófisis transversas; primera vértebra con espina neural; ausencia de arco hemal en el parahipural, centrum medio que no articula con el parahipural, patrón 6 en la fusión de hipurales; ausencia de epurales independientes, presencia de una placa que corresponde a la fusión del hipural 5+epural; espina hemal fusionada en centrum preural, presencia de infraorbitarios en el lado oculado; 13 radios ramificados en la aleta caudal; 6 radios en cada basipterigios. Sin embargo, hay caracteres que no son compartidos con este grupo, ellos serían: 17 radios en la aleta caudal más 2 radios procurrentes en cada extremo, el superior es libre y en contacto con la última espina neural, mientras que el inferior se encuentra fusionado con el último radio de la aleta.

Hensley \& Ahlstrom (1984) consideran también otros caracteres morfológicos para estructurar estos grupos, como son la posición de los ojos, abertura urinaria y ano. Por otra parte, para las familias de pleuronectiformes Ahlstrom et al. (1984) consideraron las características de los huevos y del desarrollo larval. En relación a lo anterior, H. macrops, se caracteriza por disponer los ojos en el lado izquierdo, la abertura urinaria en el lado oculado (izquierdo) y el ano en el lado ciego (derecho). En el caso de caracteres de huevo y de larvas descritas para la familia Paralichthyidae, éstas poseen un huevo con una gota oleosa y la migración del ojo comienza entre los 11 y $16,6 \mathrm{~mm}$; sin embargo, se distinguen de otras especies de la familia porque sus larvas son de mayor tamaño y no presentar radios prolongados en la aleta dorsal anterior (Landaeta et al. 2006), aspectos que también fueron reportados para Hippoglossina oblonga e H. stomata (Sumida et al. 1979, Fahay 1983).

En Paralichthyidae, Hensley \& Ahlstrom (1984) reconocen que el grupo Pseudorhombus, que agrupa a las especies más especializadas en determinados caracteres, usualmente se caracterizan por tener 17 radios en la aleta caudal, el epural fusionado con el quinto hipural y un radio procurrente que se fusiona con el radio ventral de la aleta caudal, mientras que las especies del grupo Paralichthys, tendrían aparentemente el carácter plesiomórfico de 18 radios en la aleta caudal, al menos un epural libre y un radio procurrente en el último radio ventral de la aleta caudal, que probablemente sea un vestigio de un radio perdido en el proceso de fusión con los radios 


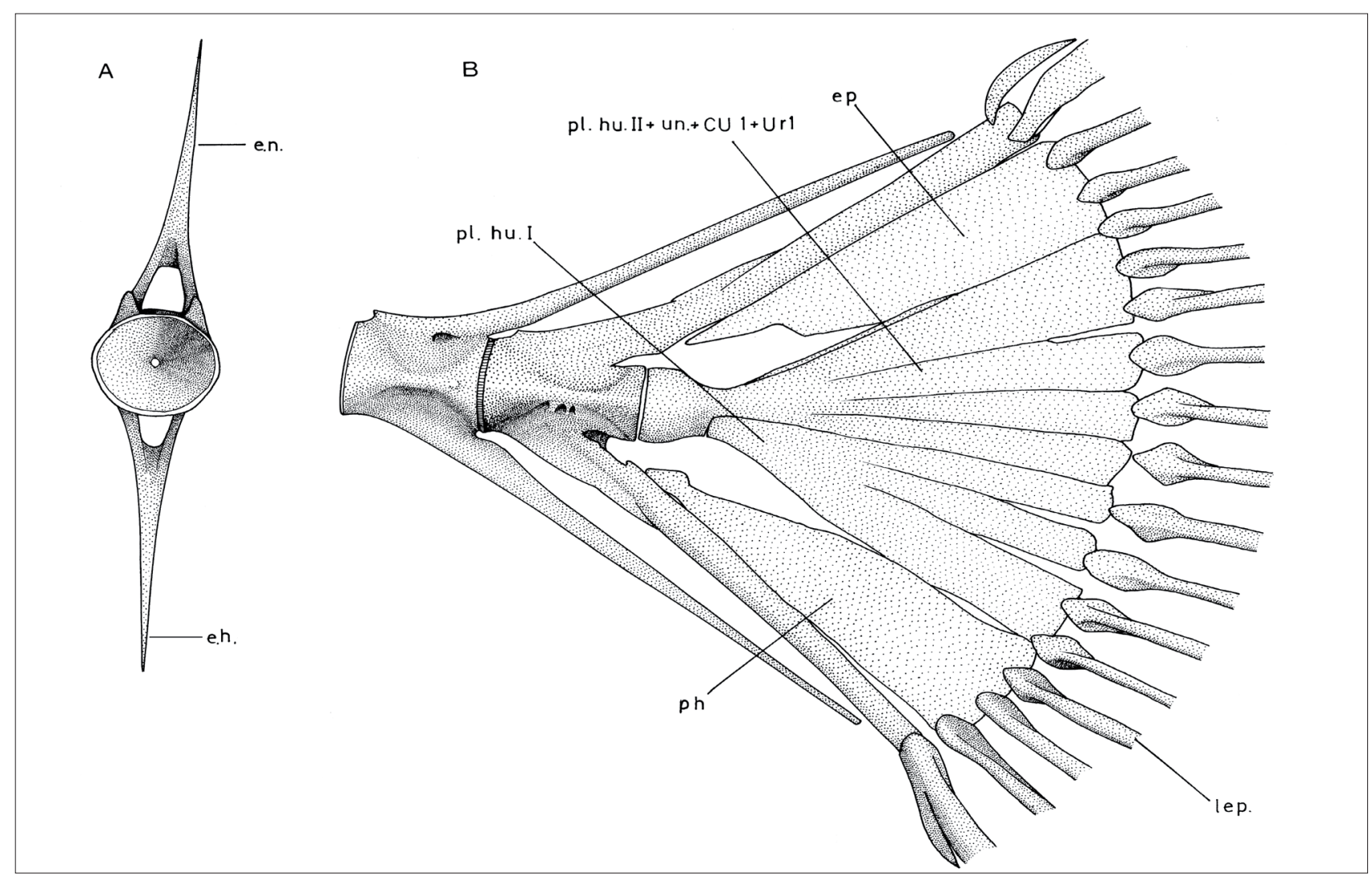

Figura 19. Hippoglossina macrops. Esqueleto caudal. A) vista anterior de la vértebra caudal y B) vista lateral del complejo hipural / Hippoglossina macrops. Caudal skeleton. A) front view of caudal vertebra and B) lateral view of the hypural complex

adyacentes. Hippoglossina macrops en base a estos tres caracteres propuestos, presenta al menos dos caracteres del grupo Pseudorhombus ( 17 radios aleta caudal +2 radios procurrentes y epural fusionado con quinto hipural) y un carácter del grupo Paralichthys como es radio procurrente que se fusiona con el último radio ventral de la aleta caudal. Esta situación lleva a plantearse preguntas sobre la posición taxonómica de Hippoglossina en la familia Paralichthyidae, especialmente su inclusión en el grupo Paralichthys propuesto por Hensley \& Ahlstrom (1984).

\section{Conclusiones}

El esqueleto óseo de Hippoglossina macrops posee en sus rasgos generales, los caracteres dados para la familia Paralichthyidae y dentro de ella comparte caracteres de los grupos Paralichthys y Pseudorhombus. En los especímenes identificados como $H$. montemaris, no se encontraron diferencias morfológicas con los especímenes estudiados de H. macrops, manteniendo la duda sobre la validez de la primera especie. El análisis de la osteología, junto a algunos caracteres morfológicos de $H$. macrops lleva a plantear la necesidad de revisar su posición, al interior de las unidades (grupos) propuestas para la familia Paralichthyidae.

\section{Agradecimientos}

Este trabajo es una deuda de mucho tiempo con quien tuvo una influencia directa en mi formación profesional, el Dr. Eduardo de la Hoz (QEPD), muchas gracias "Doc". Agradezco al Dr. Germán Pequeño, por sus siempre valiosos aportes y por la revisión de este manuscrito. También agradezco al Sr. Enrique Peralta por la confección de los dibujos.

\section{LiterATURA CITADA}

Ahlstrom EH, K Amaoka, DA Hensley, HG Moser \& BY Sumida. 1984. Pleuronectiformes: Development. En: Moser HG, WJ Richards, DM Cohen, MP Fahay, AW Kendall Jr \& SL Richardson (eds). Ontogeny and systematics of fishes. American Society of Ichthyologist and Herpetologists, Special Publication 1: 640-670.

Amaoka K. 1969. Studies on the sinistral flounders found in the waters around Japan. Taxonomy, anatomy and phylogeny. Journal of the Shimonoseki University of Fisheries 18(2): 65-340. 
Amaoka K \& DA Hensley. 2001. Paralichthyidae. Sand flounders. En: Carpenter KE \& VH Niem (eds). FAO species identification guide for fishery purposes. The living marine resources of the Western Central Pacific. Volume 6. Bony fishes part 4 (Labridae to Latimeriidae), estuarine crocodiles, sea turtles, sea snakes and marine mammals, pp. 3842-3862. FAO, Rome.

Azevedo M, C Oliveira, BG Pardo, P Martínez \& F Foresti. 2008. Phylogenetic analysis of the order Pleuronectiformes (Teleostei) based on sequences of 12S and 16S mitochondrial genes. Genetics and Molecular Biology 31(1) (suppl): 284292.

Balart E. 1985. Development of median and paired fin, skeleton of Paralichthys olivaceus (Pleuronectiformes: Paralichthyidae). Japanese Journal of Ichthyology 31(4): 398-410.

Bemvenuti M. 2005. Osteologia comparada entre as espécies de peixes-rei Odontesthes Evermann \& Kendall (Osteichthyes, Atherinopsidae) do sistema lagunar Patos-Mirim, no extremo sul do Brasil. Revista Brasileira de Zoologia 22(2): 293-305.

Berendzen P \& W Dimmick. 2002. Phylogenetic relationships of Pleuronectiformes based on molecular evidence. Copeia 2002(3): 642-652.

Bertin L \& C Arambourg. 1958. Super Ordre des Téléostéens. En: Grassé PP (ed). Traité de Zoologie 13(3): 2204-2500. Masson et $C^{\text {ie }}$ Éditeurs, Paris.

Cancino C \& F Burgos. 2009. Consideraciones ecomorfológicas del mecanismo de captura de presas en Xiphias gladius (Linnaeus, 1758). Latin American Journal of Aquatic Research 37(1): 17-28. <doi: 10.3856/vol37issue1-fulltext-2> [Special issue: Swordfish fisheries in the southeastern Pacific Ocean]

Cervigón F. 1980. Ictiología marina 1: 1-358. Editorial Arte, Caracas.

Chabanaud P. 1936. Le neurocrane osseaux des Téleosteens dyssymetriques aprés la metamorphose. Annales de l'Institut Oceanographique 16: 223-298.

Chanet B. 1994. Eubuglossus eocenicus (Woodward, 1910) from the Upper Lutetian of Egypt, one of the oldest soleids (Teleostei, Pleuronectiformes). Neues Jahrbuch für Geologie und Paläontologie-Monatshefte 7: 391-398.

Chanet B. 1995. Joleaudichthys sadeki Chabanaud, 1937: Pleuronectiform fish from egyptian Eocene. First steps on the history of Pleuronectiformes (Pisces, Teleostei). Geobios Mémoire Spécial 19: 189-191.

Chanet B. 1996a. Osteology of Solea kirchbergana (von Meyer, 1848): A soleid fish from the Lower Miocene of Germany and Switzerland. Phylogenetical implications. Neues Jahrbuch für Geologie und Palaontologie -Abhandlungen 201(1): 133-144.

Chanet B. 1996b. Oranobothus arambourgi n.g. n.sp. (Pisces Pleuronectiformes, Bothidae) provenant du Messinien
(Miocène supérieur) d'Oran (Algérie). Bulletin du Museum National d'Histoire Naturelle, Section C 18(4): 555-568.

Chanet B. 1997. A cladistic reappraisal of the fossil flatfish record consequences on the phylogeny of the Pleuronectiformes (Osteichthyes: Teleostei). Annales des Sciences Naturelles, Zoologie 18(3): 105-117.

Chapleau F. 1993. Pleuronectiform relationships: A cladistic reassessment. Bulletin of Marine Science 52(1): 516-540.

Daget J. 1964. Le crâne des téléostéens. Memoire du Museum National d'Histoire Naturelle. Nouvelle Serie. Serie A. Zoologie 31: 163-341.

De Buen F. 1961. Peces chilenos. Familias Alepocephalidae, Muraenidae, Sciaenidae, Scorpaenidae, Liparidae, Bothidae. Montemar, Chile 11(1): 1-52. [Revista de Biología Marina]

De la Hoz E. 1995. El mecanismo de mordida premaxilar en Atherinopsinae (Teleostei: Atheriniformes): aspectos estructurales relacionados con la transmisión de fuerzas. Revista Chilena de Historia Natural 68: 351-359.

De la Hoz E \& E Tosti-Croce. 1981. Osteología de Basilichthys australis Eigenmann (Teleostei-Atherinidae). Anales del Museo de Historia Natural de Valparaíso 14: 229-245.

De la Hoz E, C Cancino \& E Ojeda. 1994. Capacidades de modulación y plasticidad funcional de los mecanismos de captura de alimento en Atherinopsinae sudamericanos (Teleostei, Atherinidae). Investigaciones Marinas 22: 45-65.

Deli Antoni MY, M González \& JM Díaz de Astarloa. 2008. Análisis de la morfología ósea de peces de la laguna costera Mar Chiquita, Argentina. Revista de Biología Marina y Oceanografía 43(2): 355-380.

Díaz de Astarloa JM. 2005. Osteología craneal comparada de tres especies de lenguado del género Paralichthys (Pleuronectiformes, Paralichthyidae) del Atlántico suroccidental. Revista Chilena de Historia Natural 78(3): 343-391.

Dyer B. 2006. Systematic revision of the South American silversides (Teleostei, Atheriniformes). Biocell 30: 69-88.

Eschmeyer WN. 1998. Species in a classification. En: Eschmeyer W (ed). Catalog of fishes. Center for Biodiversity Research and Information California Academy of Sciences. Special Publication 1(3)(part.3): 2175-2443.

Evseenko SA. 1996. Ontogeny and relationships of the flatfishes of the Southern Ocean (Achiropsettidae, Pleuronectoidei). Journal of Ichthyology 36(9): 687-712.

Fahay M. 1983. Guide to the early stages of marine fishes occurring in the Western North Atlantic Ocean, Cape Hatteras to the Southern Scotian Shelf. Journal of Northwest Atlantic Fishery Science 4: 3-423.

Falabella F, R Meléndez \& ML Vargas. 1995. Claves osteológicas para peces de Chile central, un enfoque arqueológico, 208 pp. Editorial Artegrama, Santiago.

Futch C, R Topp \& E Houde. 1972. Developmental osteology of the lined sole Achirus lineatus (Pisces: Soleidae). Contribution in Marine Science 16: 33-58. 
Gosline A. 1961. The perciform caudal skeleton. Copeia 1961(3): 265-270.

Greenwood PH, DE Rosen, S Weitzman \& G Myers. 1966. Phyletic studies of teleostean fishes, with a provisional classification of living forms. Bulletin of the American Museum of Natural History 131: 341-455.

Gregory W. 1951. Evolution emerging. A survey of changing patterns from primeval life to man, $1749 \mathrm{pp}$. The Mac Millan Company, New York

Hensley DA. 1995. Paralichthyidae, Lenguados. En: Fisher W, F Krupp, W Schneider, C Sommer, KE Carpenter \& VH Niem (eds). Guía FAO para la identificación de especies para los fines de la pesca. Pacífico centro-oriental. Vol. III. Vertebrados: 1349-1380. FAO, Roma.

Hensley DA. 1997. An overview of the systematics and biogeography of the flatfishes. Journal of Sea Research 37: 187-194.

Hensley DA \& EH Ahlstrom. 1984. Pleuronectiformes: relationships. En: Moser HG, WJ Richards, DM Cohen, MP Fahay, AW Kendall Jr \& SL Richardson (eds). Ontogeny and systematics of fishes. American Society of Ichthyologist and Herpetologist, Special Publication 1: 670-687.

Hoshino K. 2001. Homologies of the caudal fin rays of Pleuronectiformes (Teleostei). Ichthyological Research 48: 231-246.

Hoshino K. 2006. Fixing the confused term "pseudomesial bar" and homologies of Pleuronectiform cranial elements, with proposals of new terms. Ichthyological Research 53: 435-440.

Hoshino K \& $K$ Amaoka. 1998. Osteology of the flounder, Tephrinectes sinensis (Lacepède) (Teleostei: Pleuronectiformes), with comments on its relationships. Ichthyological Research 45(1): 69-77.

Hosoyama K \& K Kawamura. 1993. Skeletal structure of the branchial arches in Paralichthys olivaceus (Temminck et Schlegel). Bulletin of National Research Institute of Aquaculture 22: 1-10.

Kobelkowsky DA. 2002. Osteología del lenguado Citharichthys spilopterus (Pisces: Pleuronectiformes). Anales del Instituto Biología, Universidad Nacional Autónoma de México, Serie Zoología 73(1): 53-65.

Kong I \& P Iratchet. 1995. Osteología comparada del cráneo de ocho especies de peces Sciaenidae de Chile. Estudios Oceanológicos 14: 5-16.
Landaeta MF, GA Herrera, M Pedraza, CA Bustos \& LR Castro. 2006. Reproductive tactics and larval development of bigeye flounder, Hippoglossina macrops, off central Chile. Journal of the Marine Biological Association of the United Kingdom 86: 1253-1264.

Monod T. 1968. Le complexe urophore des poissons téléostéens. Mémoires de l'Institut Fondamental d'Afrique Noire 81: 1-705.

Murakami T \& K Amaoka. 1992. Reviev of the genus Syacium (Paralichthyidae) with the description of a new species from Ecuador and Columbia. Bulletin of the Faculty of Fisheries, Hokkaido University 43(2): 61-95.

Nelson JS. 2006. Fishes of the world, 600 pp. John Wiley \& Sons, New York.

Norman J. 1934. A systematic monograph of the flatfish (Heterosomata). British Museum, London 1: 1-459.

Pardo B, A Machordom, F Foresti, F Porto-Foresti, MFC Azevedo, R Bañon, L Sanchez \& P Martínez. 2005. Phylogenetic analysis of flatfish (Order Pleuronectiformes) based on mitochondrial 16S rDNA sequences. Scientia Marina 69(4): 531-543.

Patterson C \& GD Johnson. 1995. The intermuscular bones and ligaments of teleostean fishes. Smithsonian Contributions to Zoology 559: 1-83.

Pequeño G. 1989. Peces de Chile. Lista sistemática revisada y comentada. Revista de Biología Marina 24(2): 1-132.

Pequeño G. 1997. Peces de Chile. Lista sistemática revisada y comentada: addendum. Revista de Biología Marina y Oceanografía 32(2): 77-94.

Pequeño G \& E D'Ottone. 1987. Diferenciación taxonómica de los lenguados comunes de Valdivia (Osteichthyes, Bothidae). Revista de Biología Marina 23(1): 107-137.

Sumida BY, E Ahlstrom \& G Moser. 1979. Early development of seven flatfish of the Eastern North Pacific with heavily pigmented larvae (Pisces, Pleuronectiformes). Fishery Bulletin 77(1): 105-145.

Taylor W \& G Van Dyke. 1985. Revised procedures for staining and clearing small fishes and other vertebrates for bone and cartilage study. Cybium 9(2): 107-119.

Woolcott S, C Beirne \& W Hall. 1968. Descriptive and comparative osteology of the young of the species of flounders, genus Paralichthys. Chesapeake Science 9(2): 109-120.

Recibido el 31 de agosto de 2009 y aceptado el 03 de febrero de 2010 
Anexo. Lista de abreviaturas de las figuras / Abbreviations list of the figures

$\begin{array}{ll}\text { NEUROCRÁNEO } & \\ \text { Región Etimoideal } & \\ \text { car. et. } & \text { cartílago etmoidal } \\ \text { et. lat. n. } & \text { etmoides lateral derecho } \\ \text { et. lat. z. } & \text { etmoides lateral izquierdo } \\ \text { meset. } & \text { mesetmoides } \\ \text { na. } & \text { nasal } \\ \text { pv. } & \text { prevómer } \\ \text { Región Orbital } & \\ \text { fr. n. } & \text { frontal derecho } \\ \text { fr. z. } & \text { frontal izquierdo } \\ \text { pls. } & \text { pleuroesfenoides } \\ \text { Región Oticoccipital y Basicraneal } \\ \text { boc. } & \text { basioccipital } \\ \text { epiot. } & \text { epiótico } \\ \text { esf. } & \text { esfenótico } \\ \text { exoc. } & \text { exoccipital } \\ \text { ext. } & \text { extraescapular } \\ \text { f. hm. } & \text { fosa hiomandibular } \\ \text { for. ci. } & \text { foramen carotida interna } \\ \text { for. m. } & \text { foramen magnum } \\ \text { for. V. } & \text { foramen rama oftálmica trigémino facial } \\ \text { for. VII. } & \text { foramen nervio hioideo mandibular } \\ \text { for. X } & \text { foramen del nervio vago } \\ \text { int. } & \text { intercalar } \\ \text { pa. } & \text { parietal } \\ \text { pas. } & \text { paraesfenoides } \\ \text { pro. } & \text { pterótico } \\ \text { pto. } & \text { supraoccipital } \\ \text { soc. } & \\ & \end{array}$

\section{ESPLANCNOCRÁNEO}

\begin{tabular}{ll} 
ar.an. & articular-angular \\
car. meck. & cuadrado \\
car. rost & cartílago Meckel \\
cormeck. & cartílago rostral \\
dn. & coronomeckeliano \\
ecpt. & dentario \\
entp. & ectopterigoides \\
for. lig. & entopterigoides \\
hm. & foramen ligamento \\
inf. & hiomandibular \\
iop. & infraorbitarios \\
la. & interopérculo \\
mpt. & lacrimal \\
mx. & metapterigoides \\
op. & maxila \\
pl. & opérculo \\
\hline
\end{tabular}

$\begin{array}{ll}\text { pmx. } & \text { premaxila } \\ \text { pop. } & \text { preopérculo } \\ \text { rar. } & \text { retroarticular } \\ \text { simp. } & \text { simpléctico } \\ \text { sop. } & \text { subopérculo } \\ & \\ \text { ARCO HIOIDEO } & \\ \text { bh. } & \text { basihial } \\ \text { brtg. } & \text { huesos branquiostegales } \\ \text { cha. } & \text { ceratohial anterior } \\ \text { chm. } & \text { ceratohial medio } \\ \text { chp. } & \text { ceratohial posterior } \\ \text { h.h. } & \text { hipohial } \\ \text { ih. } & \text { interhial } \\ \text { ph. } & \text { parahial }\end{array}$

\section{ARCOS BRANQUIALES}

bbr. 1, 2, 3, 4 . basibranquiales 1, 2, 3, 4 .

cbr. $1,2,3,4,5$. ceratobranquiales $1,2,3,4,5$. ebr. 1, 2, 3, 4 epibranquiales 1, 2, 3, 4 .

fbr. 1, 2, 3. faringobranquiales 1, 2, 3 .

hbr. 1, 2, $3 . \quad$ hipobranquiales 1, 2, 3.

plf. placa faríngea dentada

\section{ESQUELETO APENDICULAR}

ac.

bpt. basipterigios

cl. cleitrum

cor. coracoides

esc. escapular

pcl 1, 2. postcleitrum 1, 2.

pt. postemporal

scl. supracleitrum

\section{ESQUELETO AXIAL}

$\begin{array}{ll}\text { a. epn. } & \text { costillas epineurales } \\ \text { a. epp. } & \text { costillas epipleurales } \\ \text { c. pl. } & \text { costillas pleurales } \\ \text { e. h. } & \text { espina hemal } \\ \text { e. n. } & \text { espina neural } \\ \text { ha. } & \text { hemapófisis } \\ \text { hab } & \text { hueso abdominal } \\ \text { pti. } & \text { pterigióforo }\end{array}$

ESQUELETO CAUDAL

$\begin{array}{ll}\text { ep. } & \text { epural } \\ \text { lep. } & \text { lepidotriquias } \\ \text { ph. } & \text { parhipural } \\ \text { pl. hu. I. } & \text { placa hipural I } \\ \text { Pl. hu. II+un.+ } & \text { placa hipural II + uroneural + } \\ \text { CU1+Ur1 } & \text { centro ural 1+ ural 1 }\end{array}$

